CERN-TH.6832/93

\title{
High-Energy Approximation for On-Shell W-Pair Production ${ }^{\dagger}$
}

\author{
W. BeenakKer ${ }^{1}$, A. Denner ${ }^{2}$, S. Dittmaier $^{3}$, \\ R. MERTIG ${ }^{4}$ AND T. AACK $^{5}$
}

1 Theory Division, DESY, Hamburg, Germany

2 Theory Division, CERN, Geneva, Switzerland

${ }^{3}$ Physikalisches Institut, University of Würzburg, Germany

${ }^{4}$ Instituut Lorentz, University of Leiden, The Netherlands

5 Siemens AG, Public Telecommunications, Munich, Germany

\begin{abstract}
:
Starting from the exact virtual and soft-photonic $\mathcal{O}(\alpha)$ corrections to the differential cross-section for on-shell $\mathrm{W}$-pair production we derive a high-energy approximation valid for energies much higher than the $\mathrm{W}$-boson mass. This approximation is improved by taking into account extra terms for large top-quark and Higgs-boson masses as well as the exact results for the leading low-energy universal corrections associated with the running of $\alpha$ and the $\rho$-parameter. For $90^{\circ}$ scattering angle and in those situations where the cross-section is large, this approximation reproduces the full virtual and soft-photonic $\mathcal{O}(\alpha)$ result to better than $\sim 1 \%$ for energies above $500 \mathrm{GeV}$. In the angular range $-0.9<\cos \theta<0.9$ it is better than $1 \%$ for energies above $1-3 \mathrm{TeV}$, depending on the polarization.
\end{abstract}

CERN-TH.6832/93

March 1993

${ }^{\dagger}$ Work supported in part by the NATO Collaborative Research Grant No. CRG 900136. 


\section{Introduction}

One of the most important processes for the investigation of the Minimal Standard Model (MSM) in the future is the production of $\mathrm{W}$ pairs [1]. First of all it will allow a precise direct measurement of the mass of the $\mathrm{W}$ boson at LEP200. Even more important, it provides the first accurate direct investigation of the non-Abelian triple gauge couplings. The sensitivity to anomalous gauge couplings grows strongly with energy owing to the fact that these couplings in general spoil the unitarity cancellations for longitudinal gauge bosons present in the MSM.

The cross-section for on-shell W-pair production in the MSM is known, including the complete set of $\mathcal{O}(\alpha)$ corrections [2, 3, 4, 5]. However, these results are very lengthy and complicated, resulting in huge and rather slow computer programs. Moreover, the formulae are completely untransparent. In view of this, simple approximative expressions are desirable. This has already been accomplished for LEP200 energies [6]. For intermediate and high energies, however, there exist no reasonably simple approximations reproducing the full $\mathcal{O}(\alpha)$ results sufficiently well. In view of the expected experimental accuracy of the next linear $\mathrm{e}^{+} \mathrm{e}^{-}$colliders, approximations with an error at the level of $1 \%$ should be aimed at.

At high energies a natural approach is to neglect the fermion and boson masses as compared with the energy of the process whenever possible. For intermediate energies $500 \mathrm{GeV}<E_{\mathrm{CMS}}<2 \mathrm{TeV}$, however, this becomes far from evident, as the masses of the Higgs-boson and the top-quark can be comparable with the energy, resulting in large delayed unitarity effects [7]. Moreover even the $\mathrm{W}$ and $\mathrm{Z}$ masses may become relevant.

In this paper we present a high-energy approximation for the differential cross-section for polarized electrons and $\mathrm{W}$ bosons including virtual and soft-photonic $\mathcal{O}(\alpha)$ corrections. It is based on the assumption that while $M_{\mathrm{H}}$ and $m_{\mathrm{t}}$ are arbitrary all the Mandelstam variables $s,|t|,|u|$ are large compared with the $\mathrm{W}$-boson mass. Consequently the resulting formulae will fail in the very forward and backward directions. Since these regions of phase space will be anyhow removed by experimental cuts, our approximation should be sufficient for all practical purposes. In addition we give explicit results for four interesting special limits of our approximation: $M_{\mathrm{W}}^{2} \ll s \ll M_{\mathrm{H}}^{2}, M_{\mathrm{W}}^{2} \ll s \ll m_{\mathrm{t}}^{2}, M_{\mathrm{W}}^{2} \ll M_{\mathrm{H}}^{2} \ll s$, and $M_{\mathrm{W}}^{2} \ll m_{\mathrm{t}}^{2} \ll s$.

Our strategy for the construction of a reasonable approximation for intermediate energies is to connect the existing low-energy approximation with the high-energy approximation constructed in this paper. The interface between the two consists in exactly taking into account the leading low-energy universal corrections, i.e. the running of $\alpha$, the universal corrections $\propto m_{\mathrm{t}}^{2} / M_{\mathrm{W}}^{2}$ associated with the $\rho$-parameter, and the leading QED logarithms.

The paper is organized as follows: In Section 2 we introduce our notation and conventions. The analytic formulae for the high-energy approximation are presented and discussed in Section 3 and Appendix B. In Section 4 this approximation is refined to include the leading low-energy universal corrections exactly. The quality of the approximations is discussed numerically in Section 5. After the conclusions given in Section 6 we provide in Appendix A a detailed list of the scalar integrals needed for the high-energy approximation. 


\section{Notation and conventions}

The momenta and helicities of the incoming leptons and outgoing $\mathrm{W}$ bosons are defined through $\left(\kappa_{ \pm}=+\frac{1}{2},-\frac{1}{2} ; \lambda_{ \pm}=+1,0,-1\right)$

$$
\mathrm{e}^{+}\left(p_{+}, \kappa_{+}\right)+\mathrm{e}^{-}\left(p_{-}, \kappa_{-}\right) \rightarrow \mathrm{W}^{+}\left(k_{+}, \lambda_{+}\right)+\mathrm{W}^{-}\left(k_{-}, \lambda_{-}\right) .
$$

In the centre-of-mass system of the $\mathrm{e}^{+} \mathrm{e}^{-}$pair the momenta read

$$
p_{ \pm}=E(1,0,0, \mp 1), \quad k_{ \pm}=E(1, \mp \beta \sin \theta, 0, \mp \beta \cos \theta)
$$

where $E$ denotes the beam energy, $\theta$ the scattering angle between the $\mathrm{e}^{+}$and the $\mathrm{W}^{+}$, and $\beta=\sqrt{1-M_{\mathrm{W}}^{2} / E^{2}}$. The Mandelstam variables used in the following are given by

$$
\begin{aligned}
& s=\left(p_{+}+p_{-}\right)^{2}=\left(k_{+}+k_{-}\right)^{2}=4 E^{2}, \\
& t=\left(p_{+}-k_{+}\right)^{2}=\left(p_{-}-k_{-}\right)^{2}=-E^{2}\left(1+\beta^{2}-2 \beta \cos \theta\right), \\
& u=\left(p_{+}-k_{-}\right)^{2}=\left(p_{-}-k_{+}\right)^{2}=-E^{2}\left(1+\beta^{2}+2 \beta \cos \theta\right) .
\end{aligned}
$$

In order to define helicity amplitudes we need to introduce the corresponding polarization vectors for the $\mathrm{W}^{+}$and $\mathrm{W}^{-}$bosons

$$
\begin{aligned}
& \varepsilon_{ \pm}^{\mu}\left(k_{ \pm},+1\right)=\frac{1}{\sqrt{2}}(0, \mp \cos \theta,-i, \pm \sin \theta), \\
& \varepsilon_{ \pm}^{\mu}\left(k_{ \pm},-1\right)=\frac{1}{\sqrt{2}}(0, \mp \cos \theta,+i, \pm \sin \theta), \\
& \varepsilon_{ \pm}^{\mu}\left(k_{ \pm}, 0\right)=\frac{E}{M_{W}}(\beta, \mp \sin \theta, 0, \mp \cos \theta) .
\end{aligned}
$$

Because we are neglecting the electron mass, the helicity of the positron is opposite to the helicity of the electron

$$
\kappa_{-}=-\kappa_{+}=\kappa
$$

\section{The high-energy approximation}

We have derived a high-energy approximation to the full $\mathcal{O}(\alpha)$ virtual and softphotonic corrections for on-shell W-pair production in the MSM. It is based on the assumption that $s,|t|,|u| \gg M_{\mathrm{W}}^{2}$. As a consequence this approximation is not valid in the very forward and backward directions where $|t|$ or $|u|$ become comparable with $M_{\mathrm{W}}^{2}$. In addition we have neglected all light fermion masses $\left(m_{\mathrm{e}}, m_{\mu}, m_{\tau}, m_{\mathrm{d}}, m_{\mathrm{u}}, m_{\mathrm{s}}, m_{\mathrm{c}}, m_{\mathrm{b}}\right)$ with respect to $M_{\mathrm{W}}$ whenever possible.

The approximation has been derived using two independent methods. The first one consists in a direct expansion of the complete $\mathcal{O}(\alpha)$ formulae carried out with the help of Mathematica [8] and Form [9]. To this end the existing results have been directly reduced to scalar integrals. The coefficients accompanying these scalar functions were expanded up to the relevant order in $M_{\mathrm{W}}^{2} / x, x=s, t, u$. Subsequently approximate expressions for the scalar integrals, listed in Appendix A, have been inserted. Finally all terms that vanish with $M_{\mathrm{W}}^{2} / x$ have been discarded. 
The second method exploits the fact that in the high-energy limit the Green functions for longitudinal $\mathrm{W}$ bosons are related to the ones with external unphysical charged Higgsbosons (equivalence theorem [10]). This allows the derivation of high-energy relations between the corresponding matrix elements, from which the leading terms in a highenergy expansion for longitudinal $\mathrm{W}$ bosons can be obtained.

For large Higgs or top masses and longitudinal $\mathrm{W}$ bosons the leading terms are of the form $M_{\mathrm{H}}^{2} / M_{\mathrm{W}}^{2}\left(M_{\mathrm{H}}^{2} / s\right)^{n}, m_{\mathrm{t}}^{2} / M_{\mathrm{W}}^{2}\left(m_{\mathrm{t}}^{2} / s\right)^{n}, n=0,1,2$. Consequently there are terms of the form $\left(M_{\mathrm{H}}^{2} / s\right)^{n},\left(m_{\mathrm{t}}^{2} / s\right)^{n}, n=1,2,3$, which are suppressed compared with these leading terms but nevertheless do not vanish in the limit $M_{\mathrm{W}}^{2} \ll s$. Evidently the equivalence theorem cannot give these subleading terms. They can, however, be derived by the direct expansion. Because of the presence of these leading terms the expansion for longitudinal $\mathrm{W}$ bosons has to include terms of $\mathcal{O}\left(M_{\mathrm{W}}^{2} / s\right)$ in intermediate steps. For transverse $W$ bosons, however, all leading terms of this sort originate from the $\rho$-parameter, rendering the determination of the subleading terms trivial.

We start out by giving the high-energy approximation for the following lowest-order differential cross-sections:

$$
\begin{aligned}
& \left(\frac{d \sigma}{d \Omega}\right)_{\kappa, L}^{\text {Born }}=\frac{d \sigma}{d \Omega}^{\text {Born }}\left(\kappa, \lambda_{+}=0, \lambda_{-}=0\right), \quad\left(\frac{d \sigma}{d \Omega}\right)_{\kappa, T}^{\text {Born }}=\sum_{\lambda_{+}, \lambda_{-}= \pm 1} \frac{d \sigma^{\text {Born }}}{d \Omega}\left(\kappa, \lambda_{+}, \lambda_{-}\right), \\
& \left(\frac{d \sigma}{d \Omega}\right)_{\kappa, M}^{\text {Born }}=\sum_{\lambda_{+}= \pm 1}{\frac{d \sigma^{\text {Born }}}{d \Omega}}^{\text {( }}\left(\kappa, \lambda_{+}, \lambda_{-}=0\right)+\sum_{\lambda_{-}= \pm 1} \frac{d \sigma^{\text {Born }}}{d \Omega}\left(\kappa, \lambda_{+}=0, \lambda_{-}\right) .
\end{aligned}
$$

They read

$$
\begin{aligned}
& \left(\frac{d \sigma}{d \Omega}\right)_{-, L}^{\text {Born }}=F \frac{1}{16 s_{\mathrm{W}}^{4} c_{\mathrm{W}}^{4}} \sin ^{2} \theta, \quad\left(\frac{d \sigma}{d \Omega}\right)_{+, L}^{\text {Born }}=F \frac{1}{4 c_{\mathrm{W}}^{4}} \sin ^{2} \theta \\
& \left(\frac{d \sigma}{d \Omega}\right)_{-, T}^{\text {Born }}=F \frac{1}{4 s_{\mathrm{W}}^{4}} \frac{u^{2}+t^{2}}{t^{2}} \sin ^{2} \theta, \quad\left(\frac{d \sigma}{d \Omega}\right)_{+, T}^{\text {Born }}=F \frac{2}{c_{\mathrm{W}}^{4}} \frac{M_{\mathrm{W}}^{4}}{s^{2}} \sin ^{2} \theta \\
& \left(\frac{d \sigma}{d \Omega}\right)_{-, M}^{\text {Born }}=F \frac{1}{s_{\mathrm{W}}^{4} c_{\mathrm{W}}^{4}} \frac{M_{\mathrm{W}}^{2}}{s} \frac{u^{2}+t^{2}+4 c_{\mathrm{W}}^{2} u(u-t)+8 c_{\mathrm{W}}^{4} u^{2}}{s^{2}} \\
& \left(\frac{d \sigma}{d \Omega}\right)_{+, M}^{\text {Born }}=F \frac{4}{c_{\mathrm{W}}^{4}} \frac{M_{\mathrm{W}}^{2}}{s} \frac{u^{2}+t^{2}}{s^{2}},
\end{aligned}
$$

where

$$
F=\frac{\alpha^{2}}{4 s} \beta
$$

and

$$
c_{\mathrm{W}}=\frac{M_{\mathrm{W}}}{M_{\mathrm{Z}}}, \quad s_{\mathrm{W}}=\sqrt{1-c_{\mathrm{W}}^{2}} .
$$

As can be seen, the cross-sections $(d \sigma / d \Omega)_{ \pm, M}$ and $(d \sigma / d \Omega)_{+, T}$ are suppressed relative to the others at high energies by $M_{\mathrm{W}}^{2} / s$ and $\left(M_{\mathrm{W}}^{2} / s\right)^{2}$, respectively. In the following we will only consider the cross-sections for the non-suppressed polarizations and the unpolarized cross-section.

In the cross-sections (7) we have kept the global factor $\beta$ arising from the phase-space integration. The same conventions are adopted for the corrected cross-sections. This 
means that we construct a high-energy approximation for the matrix element squared only. Note that replacing $\beta$ by 1 would affect the subleading contributions for $M_{\mathrm{W}}^{2} \ll s \sim$ $M_{\mathrm{H}}^{2}, m_{\mathrm{t}}^{2}$. In this respect it should be noted that we use the exact form of the Mandelstam variables in all the approximations, i.e. we $\operatorname{do}$ not set $\beta$ equal to one in (3).

For the non-suppressed polarizations we give the virtual and soft-photonic $\mathcal{O}(\alpha)$ corrections in the high-energy approximation as correction factors to the lowest-order crosssections defined in (7). We list separately the correction factors for the gauge-independent contributions from bosons $C^{B}$ and fermions $C^{F}$. Furthermore we split each of them into the contributions for light Higgs and top masses $\left(C^{0}\right)$, i.e. for $M_{\mathrm{H}}^{2}, m_{\mathrm{t}}^{2} \ll s,|t|,|u|$, as well as the leading $\left(C^{1}\right)$ and the subleading $\left(C^{2}\right)$ additional contributions for large Higgs and top masses:

$$
C_{\kappa, L / T}^{B / F}=C_{\kappa, L / T}^{B / F, 0}+C_{\kappa, L / T}^{B / F, 1}+C_{\kappa, L / T}^{B / F, 2}
$$

The high-energy correction factors $C_{\kappa, L / T}^{B / F, 0}$ are separated into a dominant part, including the full energy and angular dependence present in $C^{0}$, a constant part, and a part depending on the Higgs/top mass, according to

$$
\begin{aligned}
& C_{\kappa, L / T}^{B, 0}=C_{\kappa, L / T}^{B, \text { dom }}+C_{\kappa, L / T}^{B, \text { const }}+C_{\kappa, L / T}^{\mathrm{H}}, \\
& C_{\kappa, L / T}^{F, 0}=C_{\kappa, L / T}^{F, \text { dom }}+C_{\kappa, L / T}^{F, \text { const }}+C_{\kappa, L / T}^{\mathrm{t}} .
\end{aligned}
$$

We only give here the correction factors $C_{\kappa, L / T}^{B / F, \text { dom }}$, which can become large in the highenergy limit. The remaining correction factors are listed in Appendix B.

Since we are only dealing with $\mathcal{O}(\alpha)$ corrections, it is sufficient to give only the real part of the various correction factors:

$$
\begin{aligned}
C_{-, L}^{B, \text { dom }}= & \Delta_{\mathrm{LL}}+\frac{\alpha}{2 \pi}\left[-\frac{1-2 c_{\mathrm{W}}^{2}+4 c_{\mathrm{W}}^{4}}{2 c_{\mathrm{W}}^{2} s_{\mathrm{W}}^{2}} L_{s}^{2}+\frac{23+2 c_{\mathrm{W}}^{2}-48 c_{\mathrm{W}}^{4}}{12 c_{\mathrm{W}}^{2} s_{\mathrm{W}}^{2}} L_{s}+4 \frac{c_{\mathrm{W}}^{2}}{s_{\mathrm{W}}^{2}} L_{s}\left(L_{s}-L_{t}\right)\right. \\
& +\frac{\left(1-2 c_{\mathrm{W}}^{2}\right)^{2}}{c_{\mathrm{W}}^{2} s_{\mathrm{W}}^{2}}\left[L_{s} L_{\mathrm{Z}}+\left(L_{u}-L_{t}\right)\left(L_{s}-L_{\mathrm{Z}}\right)\right]+\frac{\left(1+4 c_{\mathrm{W}}^{4}\right) s^{2}}{4 c_{\mathrm{W}}^{2} s_{\mathrm{W}}^{2} u}\left(L_{s}-L_{t}\right)^{2} \\
& \left.-\frac{s}{4 c_{\mathrm{W}}^{2} s_{\mathrm{W}}^{2} t}\left(L_{s}-L_{u}\right)^{2}-2\left(L_{u}-L_{t}\right)^{2}-8 \mathrm{Li}_{2}\left(-\frac{u}{t}\right)+\frac{1}{2 s_{\mathrm{W}}^{2}} L_{\mathrm{H}}^{2}-\frac{M_{\mathrm{H}}^{2}}{8 s_{\mathrm{W}}^{2} M_{\mathrm{W}}^{2}}\right], \\
C_{+, L}^{B, \text { dom }}= & \Delta_{\mathrm{LL}}+\frac{\alpha}{2 \pi}\left[-\frac{5-10 c_{\mathrm{W}}^{2}+8 c_{\mathrm{W}}^{4}}{4 c_{\mathrm{W}}^{2} s_{\mathrm{W}}^{2}} L_{s}^{2}+\frac{25-25 c_{\mathrm{W}}^{2}+18 c_{\mathrm{W}}^{4}}{6 c_{\mathrm{W}}^{2} s_{\mathrm{W}}^{2}} L_{s}+\frac{L_{s} L_{\mathrm{Z}}}{2 c_{\mathrm{W}}^{2} s_{\mathrm{W}}^{2}}\right. \\
& +\frac{2\left(1-2 c_{\mathrm{W}}^{2}\right)}{c_{\mathrm{W}}^{2}}\left[L_{s} L_{\mathrm{Z}}+\left(L_{u}-L_{t}\right)\left(L_{s}-L_{\mathrm{Z}}\right)\right]+\frac{s}{2 c_{\mathrm{W}}^{2} u}\left(L_{s}-L_{t}\right)^{2} \\
& \left.-\frac{s}{2 c_{\mathrm{W}}^{2} t}\left(L_{s}-L_{u}\right)^{2}-2\left(L_{u}-L_{t}\right)^{2}-8 \mathrm{Li} i_{2}\left(-\frac{u}{t}\right)+\frac{1}{2 s_{\mathrm{W}}^{2}} L_{\mathrm{H}}^{2}-\frac{M_{\mathrm{H}}^{2}}{8 s_{\mathrm{W}}^{2} M_{\mathrm{W}}^{2}}\right], \\
C_{-, T}^{B, \mathrm{dom}}= & \Delta_{\mathrm{LL}}+\frac{\alpha}{2 \pi}\left[-\frac{1+2 c_{\mathrm{W}}^{2}+8 c_{\mathrm{W}}^{4}}{4 c_{\mathrm{W}}^{2} s_{\mathrm{W}}^{2}} L_{s}^{2}+\frac{3\left(1-2 c_{\mathrm{W}}^{2}+4 c_{\mathrm{W}}^{4}\right)}{4 c_{\mathrm{W}}^{2} s_{\mathrm{W}}^{2}} L_{s}+\frac{L_{s} L_{\mathrm{Z}}}{2 c_{\mathrm{W}}^{2} s_{\mathrm{W}}^{2}}\right. \\
& +\frac{4 u+2 s}{s_{\mathrm{W}}^{2} u} L_{s}\left(L_{s}-L_{t}\right)-\frac{2\left(1-2 c_{\mathrm{W}}^{2}\right)}{s_{\mathrm{W}}^{2}}\left[L_{s} L_{\mathrm{Z}}+\left(L_{u}-L_{t}\right)\left(L_{s}-L_{\mathrm{Z}}\right)\right] \\
& -\frac{2 s}{s_{\mathrm{W}}^{2} u}\left(L_{s}-L_{t}\right)\left(L_{s}-L_{u}\right)-\left(\frac{s\left(s+3 t-6 c_{\mathrm{W}}^{2} s\right)}{4 c_{\mathrm{W}}^{2} s_{\mathrm{W}}^{2}\left(t^{2}+u^{2}\right)}+\frac{\left(1-6 c_{\mathrm{W}}^{2}\right) s}{2 c_{\mathrm{W}}^{2} s_{\mathrm{W}}^{2} u}\right)\left(L_{s}-L_{t}\right)^{2}
\end{aligned}
$$




$$
\begin{aligned}
& -\frac{3 s t}{2 s_{\mathrm{W}}^{2}\left(t^{2}+u^{2}\right)}\left(L_{s}-L_{u}\right)^{2}-2\left(L_{u}-L_{t}\right)^{2}-8 \mathrm{Li}_{2}\left(-\frac{u}{t}\right) \\
& \left.-\frac{s\left[3 s+t+6 c_{\mathrm{W}}^{2}(s+3 t)\right]}{4 c_{\mathrm{W}}^{2} s_{\mathrm{W}}^{2}\left(t^{2}+u^{2}\right)}\left(L_{s}-L_{t}\right)-\frac{\left(1-6 c_{\mathrm{W}}^{2}\right) s u}{4 c_{\mathrm{W}}^{2} s_{\mathrm{W}}^{2}\left(t^{2}+u^{2}\right)}\right] \\
C_{-, L}^{F, \text { dom }}= & 2 \Delta \alpha-2\left(c_{\mathrm{W}}^{2}-s_{\mathrm{W}}^{2}\right) \frac{c_{\mathrm{W}}^{2}}{s_{\mathrm{W}}^{2}} \Delta \rho \\
& +\frac{\alpha}{2 \pi}\left[\frac{3 m_{\mathrm{t}}^{2}}{2 s_{\mathrm{W}}^{2} M_{\mathrm{W}}^{2}}\left(L_{\mathrm{top}}-L_{s}+\frac{3}{2}\right)+\frac{3 m_{\mathrm{t}}^{2}}{4 s_{\mathrm{W}}^{2} M_{\mathrm{W}}^{2}}\left(1-2 c_{\mathrm{W}}^{2}\right)+\frac{4\left(5-10 c_{\mathrm{W}}^{2}+8 c_{\mathrm{W}}^{4}\right)}{3 c_{\mathrm{W}}^{2} s_{\mathrm{W}}^{2}} L_{s}\right], \\
C_{+, L}^{F, \text { dom }}= & 2 \Delta \alpha+\frac{\alpha}{2 \pi}\left[\frac{3 m_{\mathrm{t}}^{2}}{2 s_{\mathrm{W}}^{2} M_{\mathrm{W}}^{2}}\left(L_{\mathrm{top}}-L_{s}+\frac{3}{2}\right)+\frac{3 m_{\mathrm{t}}^{2}}{4 s_{\mathrm{W}}^{2} M_{\mathrm{W}}^{2}}+\frac{20}{3 c_{\mathrm{W}}^{2}} L_{s}\right], \\
C_{-, T}^{F, \text { dom }}= & 2 \Delta \alpha-2 \frac{c_{\mathrm{W}}^{2}}{s_{\mathrm{W}}^{2}} \Delta .
\end{aligned}
$$

In order to make the above formulae as compact as possible we have introduced the following shorthand notations:

$$
\begin{aligned}
\Delta \alpha=- & \frac{\alpha}{3 \pi}\left[\log \frac{m_{\mathrm{e}}^{2}}{M_{\mathrm{W}}^{2}}+\log \frac{m_{\mu}^{2}}{M_{\mathrm{W}}^{2}}+\log \frac{m_{\tau}^{2}}{M_{\mathrm{W}}^{2}}+\frac{4}{3}\left(\log \frac{m_{\mathrm{u}}^{2}}{M_{\mathrm{W}}^{2}}+\log \frac{m_{\mathrm{c}}^{2}}{M_{\mathrm{W}}^{2}}\right)\right. \\
& \left.+\frac{1}{3}\left(\log \frac{m_{\mathrm{d}}^{2}}{M_{\mathrm{W}}^{2}}+\log \frac{m_{\mathrm{s}}^{2}}{M_{\mathrm{W}}^{2}}+\log \frac{m_{\mathrm{b}}^{2}}{M_{\mathrm{W}}^{2}}\right)\right], \\
\Delta \rho= & \frac{\alpha}{4 \pi} \frac{3}{4 s_{\mathrm{W}}^{2}} \frac{m_{\mathrm{t}}^{2}}{M_{\mathrm{W}}^{2}}, \\
\Delta_{\mathrm{LL}}= & \frac{\alpha}{\pi}\left[\frac{3}{2} \log \frac{s}{m_{\mathrm{e}}^{2}}+2 \log \frac{\Delta E}{E}\left(\log \frac{s}{m_{\mathrm{e}}^{2}}+\log \frac{s}{M_{\mathrm{W}}^{2}}+2 \log \frac{t}{u}-2\right)\right], \\
L_{s}= & \log \frac{s}{M_{\mathrm{W}}^{2}}, \quad L_{t}=\log \frac{-t}{M_{\mathrm{W}}^{2}}, \quad L_{u}=\log \frac{-u}{M_{\mathrm{W}}^{2}}, \\
L_{\mathrm{H}}= & \log \frac{M_{\mathrm{H}}^{2}}{M_{\mathrm{W}}^{2}}, \quad L_{\mathrm{Z}}=\log \frac{M_{\mathrm{Z}}^{2}}{M_{\mathrm{W}}^{2}}, \quad L_{\mathrm{top}}=\log \frac{m_{\mathrm{t}}^{2}}{M_{\mathrm{W}}^{2}} .
\end{aligned}
$$

Despite the fact that the remaining formulae, given in Appendix B, seem rather long at first sight, they are in fact very short compared with the full expressions. In particular the expressions for the high-energy correction factors $C^{0}$ only contain one dilogarithm originating from the soft-photon radiation. The complete high-energy approximation involves only seven different dilogarithms, while the full formulae for the $\mathcal{O}(\alpha)$ corrections involve hundreds of them. As a consequence the CPU time is reduced by a factor of 50-100 for the various polarizations.

The most important corrections at high energies result from the terms proportional to the high-energy leading logarithms $L_{s}^{2}, L_{s}$ or $\Delta_{\mathrm{LL}}$. While for the polarization $(+, L)$ the angular dependence is small the terms proportional to $L_{s}\left(L_{s}-L_{t}\right)$ cause a sizeable angular dependence at high energies for $(-, L)$ and $(-, T)$ of roughly $20 \%$ at $1 \mathrm{TeV}$ and $40 \%$ at $10 \mathrm{TeV}$. The angular dependence of all the other contributions is weaker. In particular there are no poles $1 / t$ or $1 / u$, as the corresponding residues vanish in the high-energy limit owing to the relation $s+t+u=2 M_{\mathrm{W}}^{2}$. Moreover most of the terms in the correction 
factors do not depend on the scattering angle. As mentioned above all angular-dependent contributions to the high-energy correction factors $C^{0}$ have been collected in $C^{B / F, \text { dom }}$.

Furthermore $C^{B / F, \text { dom }}$ contains leading corrections, which are of the same kind as at low energies. They consist of the running of $\alpha$, the leading virtual and soft QED logarithms $\log \left(s / m_{\mathrm{e}}^{2}\right)$ and $\log (\Delta E / E)$, and terms proportional to $m_{\mathrm{t}}^{2} / M_{\mathrm{W}}^{2}$. The low-energy leading corrections have been summarized in $\Delta \alpha, \Delta \rho$ and $\Delta_{\mathrm{LL}}$ in (12) and (B.4), bearing in mind that $\Delta_{\mathrm{LL}}$ is the high-energy approximation of the exact leading QED factor

$$
\Delta_{\mathrm{LL}}^{\text {exact }}=\frac{\alpha}{\pi}\left[\frac{3}{2} \log \frac{s}{m_{\mathrm{e}}^{2}}+2 \log \frac{\Delta E}{E}\left(\log \frac{s}{m_{\mathrm{e}}^{2}}+\frac{s-2 M_{\mathrm{W}}^{2}}{s \beta} \log \frac{1+\beta}{1-\beta}+2 \log \frac{M_{\mathrm{W}}^{2}-t}{M_{\mathrm{W}}^{2}-u}-2\right)\right] .
$$

For transverse $\mathrm{W}$ bosons all high-energy contributions, resulting from large Higgs and top masses, originate from the $\rho$-parameter. In fact the Higgs- and top-mass dependence of the transverse correction factors is entirely due to renormalization effects and is hence not intrinsically different from that at LEP200. On the contrary unitarity cancellations lead to a variety of additional contributions for longitudinal $\mathrm{W}$ bosons. Their general form, given in Appendix B, contains in particular terms proportional to $\left(M_{\mathrm{H}}^{2} / M_{\mathrm{W}}^{2}\right)^{n}$, $\left(m_{\mathrm{t}}^{2} / M_{\mathrm{W}}^{2}\right)^{n}, n=1,2,3$. In the high-energy limit $s \gg M_{\mathrm{H}}^{2}, m_{\mathrm{t}}^{2} \gg M_{\mathrm{W}}^{2}$, these reduce to terms of the form $M_{\mathrm{H}}^{2} / M_{\mathrm{W}}^{2}, L_{\mathrm{H}}^{2}$ or $m_{\mathrm{t}}^{2} / M_{\mathrm{W}}^{2}$, which we have included in $C^{B / F, \text { dom }}$, and the less important terms summarized in (18) and (19).

In the limit $M_{\mathrm{W}}^{2} \ll s \ll M_{\mathrm{H}}^{2}$ all terms of the form $\left(M_{\mathrm{H}}^{2} / M_{\mathrm{W}}^{2}\right)^{n}$ with $n>0$ disappear (screening theorem [11]), and the Higgs-dependent parts of the correction factors not contained in $C^{B, \text { dom }}$ read:

$$
\begin{aligned}
& C^{B, \mathrm{H}}=\left.\left(C^{B, \mathrm{H}}+C^{B, 1}+C^{B, 2}\right)\right|_{M_{\mathrm{W}}^{2} \ll s \ll M_{\mathrm{H}}^{2}}, \\
& C_{-, L}^{B, \mathrm{H}}=\frac{\alpha}{2 \pi}\left[\frac{L_{s}^{2}}{2 s_{\mathrm{W}}^{2}}-\frac{2+27 c_{\mathrm{W}}^{2}-36 c_{\mathrm{W}}^{4}}{12 c_{\mathrm{W}}^{2} s_{\mathrm{W}}^{2}} L_{s}-2 \frac{c_{\mathrm{W}}^{2}}{s_{\mathrm{W}}^{2}} L_{s}\left(L_{s}-L_{t}\right)-\frac{c_{\mathrm{W}}^{2} s}{2 s_{\mathrm{W}}^{2} u}\left(L_{s}-L_{t}\right)^{2}\right. \\
& -\frac{1+3 c_{\mathrm{W}}^{2}-72 c_{\mathrm{W}}^{4}+160 c_{\mathrm{W}}^{6}-72 c_{\mathrm{W}}^{8}}{24 c_{\mathrm{W}}^{6} s_{\mathrm{W}}^{4}} L_{\mathrm{Z}}-\frac{1-2 c_{\mathrm{W}}^{2}}{2 s_{\mathrm{W}}^{2}} L_{x_{\mathrm{Z}}}^{2} \\
& -\frac{\left(1-2 c_{\mathrm{W}}^{2}\right)\left(1+7 c_{\mathrm{W}}^{2}-44 c_{\mathrm{W}}^{4}+36 c_{\mathrm{W}}^{6}\right)}{24 c_{\mathrm{W}}^{6} s_{\mathrm{W}}^{4}} \beta_{\mathrm{Z}} L_{x_{\mathrm{Z}}}+\frac{6-31 c_{\mathrm{W}}^{2}-114 c_{\mathrm{W}}^{4}+188 c_{\mathrm{W}}^{6}}{72 c_{\mathrm{W}}^{4} s_{\mathrm{W}}^{2}} \\
& -\frac{5\left(1-2 c_{\mathrm{W}}^{2}\right)}{s_{\mathrm{W}}^{2}} \zeta(2)-\frac{L_{\mathrm{H}}^{2}}{2 s_{\mathrm{W}}^{2}}+\frac{M_{\mathrm{H}}^{2}}{8 s_{\mathrm{W}}^{2} M_{\mathrm{W}}^{2}}+\frac{1-12 c_{\mathrm{W}}^{2}+28 c_{\mathrm{W}}^{4}}{12 c_{\mathrm{W}}^{2} s_{\mathrm{W}}^{2}} L_{\mathrm{H}} \\
& \left.+\frac{1}{144 s_{\mathrm{W}}^{2}}\left(\frac{4 c_{\mathrm{W}}^{2} s}{t}+\frac{s}{M_{\mathrm{W}}^{2}}\right)\left(6 L_{\mathrm{H}}-6 L_{s}-1\right)\right] \text {, } \\
& C_{+, L}^{B, \mathrm{H}}=\frac{\alpha}{2 \pi}\left[\frac{L_{s}^{2}}{2 s_{\mathrm{W}}^{2}}-\frac{2+31 c_{\mathrm{W}}^{2}}{12 c_{\mathrm{W}}^{2} s_{\mathrm{W}}^{2}} L_{s}-\frac{1+6 c_{\mathrm{W}}^{2}-56 c_{\mathrm{W}}^{4}}{24 c_{\mathrm{W}}^{6} s_{\mathrm{W}}^{2}} L_{\mathrm{Z}}-\frac{L_{x_{\mathrm{Z}}}^{2}}{2 s_{\mathrm{W}}^{2}}\right. \\
& -\frac{1+8 c_{\mathrm{W}}^{2}-36 c_{\mathrm{W}}^{4}}{24 c_{\mathrm{W}}^{6} s_{\mathrm{W}}^{2}} \beta_{\mathrm{Z}} L_{x_{\mathrm{Z}}}+\frac{6-19 c_{\mathrm{W}}^{2}-78 c_{\mathrm{W}}^{4}}{72 c_{\mathrm{W}}^{4} s_{\mathrm{W}}^{2}}-\frac{5}{s_{\mathrm{W}}^{2}} \zeta(2)-\frac{L_{\mathrm{H}}^{2}}{2 s_{\mathrm{W}}^{2}}+\frac{M_{\mathrm{H}}^{2}}{8 s_{\mathrm{W}}^{2} M_{\mathrm{W}}^{2}} \\
& \left.+\frac{L_{\mathrm{H}}}{12 c_{\mathrm{W}}^{2} s_{\mathrm{W}}^{2}}+\frac{s}{144 s_{\mathrm{W}}^{2} M_{\mathrm{W}}^{2}}\left(6 L_{\mathrm{H}}-6 L_{s}-1\right)\right] \text {, } \\
& C_{-, T}^{B, H}=\frac{\alpha}{2 \pi}\left[-\frac{5}{6 s_{\mathrm{W}}^{4}} L_{\mathrm{Z}}+\frac{11}{12 s_{\mathrm{W}}^{2}} L_{\mathrm{H}}+\frac{7}{72 s_{\mathrm{W}}^{2}}\right] \text {, }
\end{aligned}
$$


with

$$
\beta_{\mathrm{Z}}=\sqrt{1-4 \frac{M_{\mathrm{W}}^{2}}{M_{\mathrm{Z}}^{2}-i \varepsilon}}, \quad x_{\mathrm{Z}}=\frac{1-\beta_{\mathrm{Z}}}{1+\beta_{\mathrm{Z}}}, \quad L_{x_{\mathrm{Z}}}=\log x_{\mathrm{Z}} .
$$

In the corresponding limit $M_{\mathrm{W}}^{2} \ll s \ll m_{\mathrm{t}}^{2}$ the top-mass-dependent parts of the correction factors not contained in $C^{F, \text { dom }}$ are given by:

$$
\begin{aligned}
C^{\prime F, \mathrm{t}}= & \left.\left(C^{F, \mathrm{t}}+C^{F, 1}+C^{F, 2}\right)\right|_{M_{\mathrm{W}}^{2} \ll s \ll m_{\mathrm{t}}^{2}}, \\
C_{-, L}^{\prime F, \mathrm{t}}= & -2 \frac{M_{\mathrm{W}}^{2}}{s} \frac{c_{\mathrm{W}}^{2}}{s_{\mathrm{W}}^{2}} \Delta \rho\left(24 c_{\mathrm{W}}^{4}+4 s_{\mathrm{W}}^{2}-\frac{2}{c_{\mathrm{W}}^{2}}+8 c_{\mathrm{W}}^{4} \frac{s}{t}\right) \\
& +\frac{\alpha}{2 \pi}\left[-\frac{11-16 c_{\mathrm{W}}^{2}-4 c_{\mathrm{W}}^{4}+144 c_{\mathrm{W}}^{6}}{18 c_{\mathrm{W}}^{2} s_{\mathrm{W}}^{2}} L_{s}+\frac{48-88 c_{\mathrm{W}}^{2}+287 c_{\mathrm{W}}^{4}+86 c_{\mathrm{W}}^{6}-360 c_{\mathrm{W}}^{8}}{108 c_{\mathrm{W}}^{2} s_{\mathrm{W}}^{4}}\right. \\
& -\frac{\left(1-4 c_{\mathrm{W}}^{2}\right)\left(2-3 c_{\mathrm{W}}^{2}+12 c_{\mathrm{W}}^{4} s_{\mathrm{W}}^{2}\right)}{6 c_{\mathrm{W}}^{2} s_{\mathrm{W}}^{4}} L_{\mathrm{top}}-\frac{3 m_{\mathrm{t}}^{2}}{2 s_{\mathrm{W}}^{2} M_{\mathrm{W}}^{2}}\left(L_{\mathrm{top}}-L_{s}+2-c_{\mathrm{W}}^{2}\right) \\
& \left.+\left(\frac{4+5 c_{\mathrm{W}}^{2}}{18 s_{\mathrm{W}}^{2}}-\frac{1-4 c_{\mathrm{W}}^{2}}{6 s_{\mathrm{W}}^{2}}\left(L_{\mathrm{top}}-L_{s}\right)\right)\left(\frac{s}{M_{\mathrm{W}}^{2}}+4 c_{\mathrm{W}}^{2} \frac{s}{t}\right)\right] \\
C_{+, L}^{\prime F, \mathrm{t}}= & \frac{\alpha}{2 \pi}\left[-\frac{11-26 c_{\mathrm{W}}^{2}}{18 c_{\mathrm{W}}^{2} s_{\mathrm{W}}^{2}} L_{s}-\frac{L_{\mathrm{top}}}{3 c_{\mathrm{W}}^{2} s_{\mathrm{W}}^{2}}+\frac{48+23 c_{\mathrm{W}}^{2}-320 c_{\mathrm{W}}^{4}}{108 c_{\mathrm{W}}^{2} s_{\mathrm{W}}^{2}}\right. \\
& \left.-\frac{3 m_{\mathrm{t}}^{2}}{2 s_{\mathrm{W}}^{2} M_{\mathrm{W}}^{2}}\left(L_{\mathrm{top}}-L_{s}+2\right)+\frac{s}{18 s_{\mathrm{W}}^{2} M_{\mathrm{W}}^{2}}\left(4+3 L_{s}-3 L_{\mathrm{top}}\right)\right] \\
C_{-, T}^{\prime F, \mathrm{t}}= & -8 \frac{M_{\mathrm{W}}^{2}}{s} \frac{c_{\mathrm{W}}^{2}}{s_{\mathrm{W}}^{2}} \Delta \rho+\frac{\alpha}{2 \pi}\left[\frac{233-580 c_{\mathrm{W}}^{2}+320 c_{\mathrm{W}}^{4}}{108 s_{\mathrm{W}}^{4}}+\frac{1-4 c_{\mathrm{W}}^{2}}{6 s_{\mathrm{W}}^{4}} L_{\mathrm{top}}\right] .
\end{aligned}
$$

By adding the fermionic correction factors of (12) and (17), one observes that the terms proportional to $\left(m_{\mathrm{t}}^{2} / M_{\mathrm{W}}^{2}\right)^{n}$ disappear for $n=2,3$ and that the remaining terms for $n=1$ are exactly the (low-energy) ones contained in $\Delta \rho$. Moreover, when combining all leading correction factors from (12) with the results from (15) and (17), also the terms involving $L_{\text {top }}$ and $L_{\mathrm{H}}$ are the same as at low energy, including those terms that are accompanied by a factor $s / M_{\mathrm{W}}^{2}[6,12]$. The latter lead to large corrections, known as delayed unitarity effects [7], and result from the fact that the unitarity cancellations are not yet complete in the presence of heavy particles.

In the limit $s \gg m_{\mathrm{t}}^{2}, M_{\mathrm{H}}^{2}$ these delayed unitarity terms disappear. On the other hand for $M_{\mathrm{W}}^{2} \ll M_{\mathrm{H}}^{2} \ll s$ screening is not complete, resulting in large corrections proportional to $M_{\mathrm{H}}^{2} / M_{\mathrm{W}}^{2}$ (see (12)) and terms containing $L_{\mathrm{H}}$ deviating from the ones appearing at low energies. As the correction factors $C^{1}$ and $C^{2}$ vanish in this limit, the nonleading Higgs-mass-dependent parts of the correction factors simplify to:

$$
\begin{aligned}
C^{\prime \prime B, H} & =\left.C^{B, \mathrm{H}}\right|_{M_{\mathrm{W}}^{2} \ll M_{\mathrm{H}}^{2} \ll s}, \\
C_{-, L}^{\prime \prime B, H} & =\frac{\alpha}{2 \pi}\left[\frac{5\left(1-2 c_{\mathrm{W}}^{2}\right)}{6 c_{\mathrm{W}}^{2} s_{\mathrm{W}}^{4}} L_{\mathrm{Z}}-\frac{10-11 c_{\mathrm{W}}^{2}}{12 c_{\mathrm{W}}^{2} s_{\mathrm{W}}^{2}} L_{\mathrm{H}}-\frac{74-7 c_{\mathrm{W}}^{2}}{72 c_{\mathrm{W}}^{2} s_{\mathrm{W}}^{2}}\right], \\
C_{+, L}^{\prime \prime B, H} & =\frac{\alpha}{2 \pi}\left[\frac{5}{6 c_{\mathrm{W}}^{2} s_{\mathrm{W}}^{2}} L_{\mathrm{Z}}-\frac{10-c_{\mathrm{W}}^{2}}{12 c_{\mathrm{W}}^{2} s_{\mathrm{W}}^{2}} L_{\mathrm{H}}-\frac{74+67 c_{\mathrm{W}}^{2}}{72 c_{\mathrm{W}}^{2} s_{\mathrm{W}}^{2}}\right],
\end{aligned}
$$




$$
C_{-, T}^{\prime B, \mathrm{H}}=C_{-, T}^{B, \mathrm{H}}
$$

Also for the fermionic correction factors the terms involving $m_{\mathrm{t}}^{2} / M_{\mathrm{W}}^{2}$ or $L_{\text {top }}$ are different in the limit $M_{\mathrm{W}}^{2} \ll m_{\mathrm{t}}^{2} \ll s$ as compared with the large top-mass limit:

$$
\begin{aligned}
& C^{\prime \prime F, \mathrm{t}}=\left.C^{F, \mathrm{t}}\right|_{M_{\mathrm{W}}^{2} \ll m_{\mathrm{t}}^{2} \ll s^{\prime}} \\
& C_{-, L}^{\prime \prime F, \mathrm{t}}=\frac{\alpha}{2 \pi}\left[-\frac{17-60 c_{\mathrm{W}}^{2}+84 c_{\mathrm{W}}^{4}-32 c_{\mathrm{W}}^{6}}{18 c_{\mathrm{W}}^{2} s_{\mathrm{W}}^{4}} L_{\mathrm{top}}-\frac{197-830 c_{\mathrm{W}}^{2}+1300 c_{\mathrm{W}}^{4}-640 c_{\mathrm{W}}^{6}}{108 c_{\mathrm{W}}^{2} s_{\mathrm{W}}^{4}}\right], \\
& C_{+, L}^{\prime \prime F, \mathrm{t}}=\frac{\alpha}{2 \pi}\left[-\frac{17-26 c_{\mathrm{W}}^{2}}{18 c_{\mathrm{W}}^{2} s_{\mathrm{W}}^{2}} L_{\mathrm{top}}-\frac{197-436 c_{\mathrm{W}}^{2}+320 c_{\mathrm{W}}^{4}}{108 c_{\mathrm{W}}^{2} s_{\mathrm{W}}^{2}}\right], \\
& C_{-, T}^{\prime \prime F, \mathrm{t}}=\frac{\alpha}{2 \pi}\left[\frac{1-4 c_{\mathrm{W}}^{2}}{6 s_{\mathrm{W}}^{4}} L_{\mathrm{top}}+\frac{233-580 c_{\mathrm{W}}^{2}+320 c_{\mathrm{W}}^{4}}{108 s_{\mathrm{W}}^{4}}\right] .
\end{aligned}
$$

When combining the leading fermionic correction factors from (12) with the results from (19), most of the $m_{\mathrm{t}}^{2} / M_{\mathrm{W}}^{2}$ terms can be associated via the equivalence theorem with $\Delta \rho$ effects in the cross-section for unphysical charged-Higgs-pair production $\left(\mathrm{e}^{+} \mathrm{e}^{-} \rightarrow\right.$ $\left.\phi^{+} \phi^{-}\right)$. Using the concept of running couplings also most of the $L_{\mathrm{top}}$-dependent terms can be obtained from this process in the same way [13]. The exception is the term

$$
\frac{\alpha}{2 \pi} \frac{3 m_{\mathrm{t}}^{2}}{2 s_{\mathrm{W}}^{2} M_{\mathrm{W}}^{2}}\left[L_{\mathrm{top}}-L_{s}+\frac{3}{2}\right],
$$

appearing both in $C_{+, L}^{F, \text { dom }}$ and $C_{-, L}^{F, \text { dom }}$, which does not originate from running couplings but results from diagrams involving enhanced Yukawa couplings. Applying the concept of running couplings to $\mathrm{e}^{+} \mathrm{e}^{-} \rightarrow \mathrm{W}^{+} \mathrm{W}^{-}$yields only the leading terms in the low-energy limit. For longitudinal $\mathrm{W}$ bosons the additional terms arise from the fact that also the masses become relevant. While normally the masses can be neglected in a strict highenergy approximation, they determine the leading behaviour in the presence of unitarity cancellations.

So far we have given the correction factors to the non-suppressed cross-sections. We will not do the same for the cross-sections $(d \sigma / d \Omega)_{+, T}$ and $(d \sigma / d \Omega)_{\kappa, M}$ as they are suppressed at high energies. As observed in the case of purely longitudinally polarized $\mathrm{W}$ bosons, however, enhanced corrections emerge in the limit $M_{\mathrm{W}}^{2} \ll s \sim m_{\mathrm{t}}^{2}, M_{\mathrm{H}}^{2}$ for the mixed polarization. The corresponding contributions to the cross-section are of the same order as the contributions to the non-suppressed polarizations summarized in $C^{2}$. In the case of transverse $W$ bosons and right-handed electrons, there are no such enhanced corrections. In order to construct an approximation for the cross-section for unpolarized W bosons with the same quality as for the leading polarizations, we are led to include the above-mentioned enhanced contributions for the mixed polarization:

$$
\begin{aligned}
\left(\frac{d \sigma}{d \Omega}\right)_{\kappa, U}= & \left(\frac{d \sigma}{d \Omega}\right)_{\kappa, L}^{\text {Born }}\left[1+C_{\kappa, L}^{B}+C_{\kappa, L}^{F}\right]+\delta_{\kappa-}\left(\frac{d \sigma}{d \Omega}\right)_{\kappa, T}^{\text {Born }}\left[1+C_{\kappa, T}^{B}+C_{\kappa, T}^{F}\right] \\
& +\left(\frac{d \sigma}{d \Omega}\right)_{\kappa, M}^{B}+\left(\frac{d \sigma}{d \Omega}\right)_{\kappa, M}^{F},
\end{aligned}
$$

with $(d \sigma / d \Omega)_{\kappa, M}^{B}$ and $(d \sigma / d \Omega)_{\kappa, M}^{F}$ as given in Appendix B. 


\section{Improvement of the approximation for the intermediate-energy range}

The approximation we have constructed so far has been derived for high energies. However, it turned out that a substantial part of the dominant corrections at high energies are the same as at low energies. Moreover these leading low-energy corrections appear as universal factors. Therefore we can easily improve our approximation by using the complete lowest-order cross-section as far as these leading corrections are concerned. To this end we discard the terms proportional to $\Delta \alpha, \Delta \rho$ and $\Delta_{\mathrm{LL}}$ in the high-energy expansion and add the exact contribution of these terms, which are obtained from the following improved Born cross-sections [6]:

$$
\begin{aligned}
{\frac{d \sigma^{\mathrm{impr}}}{d \Omega}\left(\kappa, \lambda_{+}, \lambda_{-}\right)=} & \delta_{\kappa-}\left(\frac{1}{2 s_{\mathrm{W}}^{2}}\right)^{2}\left(\frac{d \sigma}{d \Omega}\right)^{I I}\left(\kappa, \lambda_{+}, \lambda_{-}\right)\left(1+\Delta_{\mathrm{LL}}^{\text {exact }}+2 \Delta \alpha-2 \frac{c_{\mathrm{W}}^{2}}{s_{\mathrm{W}}^{2}} \Delta \rho\right) \\
& +\delta_{\kappa-} \frac{1}{2 s_{\mathrm{W}}^{2}}\left(\frac{d \sigma}{d \Omega}\right)^{I Q}\left(\kappa, \lambda_{+}, \lambda_{-}\right)\left(1+\Delta_{\mathrm{LL}}^{\text {exact }}+2 \Delta \alpha-\frac{c_{\mathrm{W}}^{2}}{s_{\mathrm{W}}^{2}} \Delta \rho\right) \\
& +\left(\frac{d \sigma}{d \Omega}\right)^{Q Q}\left(\kappa, \lambda_{+}, \lambda_{-}\right)\left(1+\Delta_{\mathrm{LL}}^{\text {exact }}+2 \Delta \alpha\right) .
\end{aligned}
$$

The relevant cross-sections are obtained from

$$
\begin{aligned}
& \left(\frac{d \sigma}{d \Omega}\right)^{I I}=\left(\frac{d \sigma}{d \Omega}\right)^{Q Q}+\left(\frac{d \sigma}{d \Omega}\right)^{t t}-\left(\frac{d \sigma}{d \Omega}\right)^{t Q}, \\
& \left(\frac{d \sigma}{d \Omega}\right)^{I Q}=-2\left(\frac{d \sigma}{d \Omega}\right)^{Q Q}+\left(\frac{d \sigma}{d \Omega}\right)^{t Q},
\end{aligned}
$$

with

$$
\begin{aligned}
& \left(\frac{d \sigma}{d \Omega}\right)_{\kappa L}^{Q Q}=F\left(\frac{s+2 M_{\mathrm{W}}^{2}}{2 M_{\mathrm{W}}^{2}} \frac{M_{\mathrm{Z}}^{2}}{s-M_{\mathrm{Z}}^{2}}\right)^{2} \frac{s-4 M_{\mathrm{W}}^{2}}{s} \sin ^{2} \theta \\
& \left(\frac{d \sigma}{d \Omega}\right)_{\kappa L}^{t Q}=2 F\left(\frac{s+2 M_{\mathrm{W}}^{2}}{2 M_{W}^{2}} \frac{M_{\mathrm{Z}}^{2}}{s-M_{\mathrm{Z}}^{2}}\right)\left(1+\frac{4 M_{\mathrm{W}}^{2}}{s}+\frac{2 M_{\mathrm{W}}^{2}}{t}\right) \sin ^{2} \theta \\
& \left(\frac{d \sigma}{d \Omega}\right)_{\kappa L}^{t t}=F\left(1+\frac{4 M_{\mathrm{W}}^{2}}{s}+\frac{2 M_{\mathrm{W}}^{2}}{t}\right)^{2} \frac{s}{s-4 M_{\mathrm{W}}^{2}} \sin ^{2} \theta \\
& \left(\frac{d \sigma}{d \Omega}\right)_{\kappa T}^{Q Q}=2 F\left(\frac{M_{Z}^{2}}{s-M_{\mathrm{Z}}^{2}}\right)^{2} \frac{s-4 M_{\mathrm{W}}^{2}}{s} \sin ^{2} \theta \\
& \left(\frac{d \sigma}{d \Omega}\right)_{\kappa T}^{t Q}=4 F\left(\frac{M_{Z}^{2}}{s-M_{\mathrm{Z}}^{2}}\right)\left(\frac{4 M_{\mathrm{W}}^{2}}{s}+\frac{M_{\mathrm{W}}^{2}}{t}\right) \sin ^{2} \theta \\
& \left(\frac{d \sigma}{d \Omega}\right)_{\kappa T}^{t t}=F \frac{\left(t^{2}+u^{2}\right) s^{2}+16 M_{\mathrm{W}}^{4} t(2 t+s)}{t^{2} s\left(s-4 M_{\mathrm{W}}^{2}\right)} \sin ^{2} \theta \\
& \left(\frac{d \sigma}{d \Omega}\right)_{\kappa M}^{Q Q}=4 F \frac{M_{\mathrm{W}}^{2}}{s} \frac{M_{\mathrm{Z}}^{4}}{M_{\mathrm{W}}^{4}} \frac{u^{2}+t^{2}-2 M_{\mathrm{W}}^{4}}{\left(s-M_{\mathrm{Z}}^{2}\right)^{2}}
\end{aligned}
$$




$$
\begin{aligned}
\left(\frac{d \sigma}{d \Omega}\right)_{\kappa M}^{t Q}=16 F & \left(\frac{M_{\mathrm{Z}}^{2}}{s-M_{\mathrm{Z}}^{2}}\right) \frac{s}{s-4 M_{\mathrm{W}}^{2}} \\
& \times \frac{1}{s^{2} t}\left[t\left(t^{2}+2 u^{2}-u t\right)+M_{\mathrm{W}}^{2}\left(u^{2}+t^{2}\right)-M_{\mathrm{W}}^{4}(u+t)-2 M_{\mathrm{W}}^{6}\right], \\
\left(\frac{d \sigma}{d \Omega}\right)_{\kappa M}^{t t}=16 F & \frac{M_{\mathrm{W}}^{2}}{s}\left(\frac{s}{s-4 M_{\mathrm{W}}^{2}}\right)^{2} \frac{1}{s^{2} t^{2}}\left[t^{2}\left(t^{2}+5 u^{2}-2 u t\right)+2 M_{\mathrm{W}}^{2} t\left(t^{2}-t u+2 u^{2}\right)\right. \\
& \left.+M_{\mathrm{W}}^{4}\left(u^{2}+t^{2}-6 u t\right)-2 M_{\mathrm{W}}^{6}(u+t)\right] .
\end{aligned}
$$

\section{$5 \quad$ Numerical evaluation}

For the numerical evaluations we used the following set of parameters:

$$
\begin{array}{llll}
\alpha=1 / 137.0359895 & G_{\mu}=1.166370 \times 10^{-5} \mathrm{GeV}^{-2}, & \\
M_{\mathrm{Z}}=91.175 \mathrm{GeV}, & M_{\mathrm{H}}=100 \mathrm{GeV}, & \\
m_{\mathrm{e}}=0.51099906 \mathrm{MeV}, & m_{\mu}=105.65839 \mathrm{MeV}, & m_{\tau}=1.7841 \mathrm{GeV}, \\
m_{\mathrm{u}}=46.0 \mathrm{MeV}, & m_{\mathrm{c}}=1.50 \mathrm{GeV}, & m_{\mathrm{t}}=140 \mathrm{GeV}, \\
m_{\mathrm{d}}=46.0 \mathrm{MeV}, & m_{\mathrm{s}}=150 \mathrm{MeV}, & m_{\mathrm{b}}=4.50 \mathrm{GeV}
\end{array}
$$

The masses of the light quarks are adjusted in such a way that the experimentally measured hadronic vacuum polarization is reproduced [14]. They only enter the quantity $\Delta \alpha$. As the Fermi constant $G_{\mu}$ is empirically much better known than the W mass, we calculate $M_{\mathrm{W}}$ from all the other parameters using the muon decay width including radiative corrections. The above set of parameters yields

$$
M_{\mathrm{W}}=80.232 \mathrm{GeV} .
$$

The cut-off energy $\Delta E$ for the soft-photon bremsstrahlung is chosen to be

$$
\Delta E=E .
$$

In that way the cut-off-dependent terms drop out and do not influence the results. For realistic situations the contribution of hard photons has to be added and the cut-off has to be adjusted in the proper way.

The lowest-order cross-sections integrated over the angular region $-0.9 \leq \cos \theta \leq 0.9$ are listed in Table 1 for the non-suppressed polarizations at various energies. Note that we applied the spin average factor $1 / 4$ also to the polarized cross-sections to account for reduced luminosity and incomplete polarization. For the integrated luminosity of $10 \mathrm{fb}^{-1}$ envisaged for a $500 \mathrm{GeV} \mathrm{e} \mathrm{e}^{-}$collider, this corresponds to $22000,20000,850$ and 180 events for $\sigma_{U}, \sigma_{-, T}, \sigma_{-, L}$ and $\sigma_{+, L}$, yielding a statistical accuracy of about $0.7 \%, 0.7 \%$, $3.4 \%$ and $7.4 \%$, respectively. As the luminosity at higher energies should be such as to compensate the drop in cross-section, these numbers for the statistical accuracy should be more or less the same at higher energies. Based on these statistical errors we take $1 \%$ as some characteristic accuracy in the following. Table 1 also shows the absolute virtual and soft-photonic $\mathcal{O}(\alpha)$ corrections as calculated from the exact formulae and from our 


\begin{tabular}{|c|c|c|c|c|}
\hline$\sqrt{s} / \mathrm{TeV}$ & 0.5 & 1 & 2 & 10 \\
\hline$\left(\sigma_{\text {exact }}^{\text {Born }+\delta \sigma_{\text {app }}}+\delta \sigma_{U} / \mathrm{fb}\right.$ & $2195_{+229}^{+224}$ & $511_{+12}^{+11}$ & $125_{-14}^{-14}$ & $4.98_{-2.99}^{-2.99}$ \\
\hline 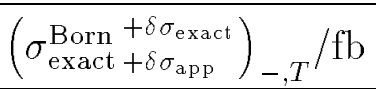 & $1966_{+196}^{+206}$ & $477_{+8}^{+7}$ & $118_{-14}^{-14}$ & $4.71_{-2.93}^{-2.93}$ \\
\hline$\left(\sigma_{\text {exact }}^{\text {Born }}+\delta \sigma_{\text {exact }}\right)_{-, L} / \mathrm{fb}$ & $85.4_{+14.2}^{+13.0}$ & $21.7_{+2.3}^{+2.2}$ & $5.46_{+0.13}^{+0.11}$ & $0.219_{-0.058}^{-0.058}$ \\
\hline$\left(\sigma_{\text {exact }}^{\text {Born }+\delta \sigma_{\text {exact }}}+\delta \sigma_{\text {app }}\right)_{+, L} / \mathrm{fb}$ & $17.9_{+5.3}^{+5.2}$ & $4.47_{+1.15}^{+1.15}$ & $1.12_{+0.24}^{+0.24}$ & $0.0446_{+0.0028}^{+0.0028}$ \\
\hline
\end{tabular}

Table 1: Lowest-order cross-sections integrated over $-0.9 \leq \cos \theta \leq 0.9$ together with the exact and approximated $\mathcal{O}(\alpha)$ corrections.

approximation. Note that the relative corrections in general grow with energy, e.g. for the unpolarized cross-section from $10 \%$ at $0.5 \mathrm{TeV}$ to $60 \%$ at $10 \mathrm{TeV}$.

For the discussion of the quality of our approximations, we need to compare with the full $\mathcal{O}(\alpha)$ corrections using a sensible normalization. For unpolarized W bosons one can simply use the lowest-order cross-section. In the case of longitudinal W bosons and left-handed electrons, however, the presence of a zero in the matrix element (see Fig. 2) makes the corresponding cross-section useless as normalization. For polarized $\mathrm{W}$ bosons we therefore prefer to use the high-energy approximations (7) of the lowest-order crosssections. Since for longitudinal $\mathrm{W}$ bosons and left-handed electrons the $t$-channel pole drops out at high energies, but is sizeable at small and intermediate energies, we add the leading $t$-channel contribution and use

$$
\left(\frac{d \sigma}{d \Omega}\right)_{-, L}^{\mathrm{int}}=\frac{F \sin ^{2} \theta}{16 s_{\mathrm{W}}^{4} c_{\mathrm{W}}^{4}}\left[1+16 c_{\mathrm{W}}^{4} \frac{M_{\mathrm{W}}^{4}}{t^{2}}\right]
$$

as normalization. Accordingly, the quantities $\Delta^{\text {Born }}, \Delta$ and $\Delta^{\prime}$ shown in the following plots are defined as

$$
\Delta^{\text {Born }}=\left|\frac{\sigma_{\text {app }}^{\text {Born }}-\sigma_{\text {exact }}^{\text {Born }}}{\sigma_{\text {exact }}^{\text {Born }}}\right|, \quad \Delta=\left|\frac{\delta \sigma_{\text {app }}-\delta \sigma_{\text {exact }}}{\sigma_{\text {exact }}^{\text {Born }}}\right|, \quad \Delta^{\prime}=\left|\frac{\delta \sigma_{\text {app }}-\delta \sigma_{\text {exact }}}{\sigma_{\text {app }}^{\text {Born }}}\right|,
$$

where $\delta \sigma_{\text {exact }}$ is the full $\mathcal{O}(\alpha)$ correction, $\delta \sigma_{\text {app }}$ the corresponding high-energy approxima-

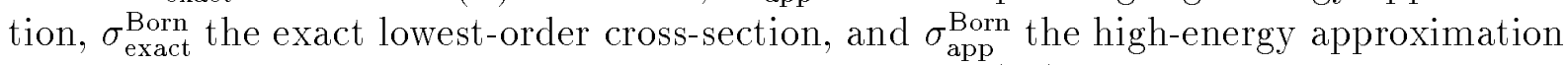
for the lowest-order cross-section modified according to (24).

In Fig. 1 we compare the relevant lowest-order approximations (7), including the modification (24), with the corresponding exact lowest-order cross-sections as a function of energy at different scattering angles. In agreement with $(23)$ we observe that for $(d \sigma / d \Omega)_{+, L}^{\text {Born }}$ the deviation between the approximation and the exact lowest-order result is angularindependent. For $(d \sigma / d \Omega)_{-, T}^{\text {Born }}$ the deviation is just weakly depending on the scattering angle. The dips in the curve for $(d \sigma / d \Omega)_{-, L}^{\text {Born }}$ are due to a cross-over of the approximation and the exact result, which is blown up by the logarithmic scale used in Fig. 1. This is a phenomenon that will show up frequently in the forthcoming plots and that will overrate the quality of the corresponding approximation. The huge errors for $\theta=25^{\circ}$ 
between $400 \mathrm{GeV}$ and $1 \mathrm{TeV}$ are a result of the above-discussed zero of the lowest-order cross-section $(d \sigma / d \Omega)_{-. L}^{\text {Born }}$. From Fig. 2 we see that the approximation (24) interpolates nicely between low and high energies, avoiding the zero.

Figure 1 also gives an indication of the quality we might expect of a high-energy approximation. More precisely, the relative deviations between approximation and exact result that we observe for the lowest-order cross-section indicate how good the approximation for the corrections can be as compared with the exact $\mathcal{O}(\alpha)$ corrections. The relative deviation at lowest order is easily estimated as $4 M_{\mathrm{W}}^{2} / s$ for the polarization $(-, T), 2 M_{\mathrm{Z}}^{2} / s$ for $(+, L)$, and $8 c_{\mathrm{W}}^{2} M_{\mathrm{W}}^{2} / t+\left(2 / c_{\mathrm{W}}^{2}-4+24 c_{\mathrm{W}}^{2}\right) M_{\mathrm{W}}^{2} / s$ for $(-, L)$. Accordingly the approximation typically reaches $1 \%$ at $2 \mathrm{TeV}$. The strikingly larger deviation for $(-, L)$ at $25^{\circ}$ even in this energy range is due to the $1 / t$ pole, which leads to an enhancement factor $s / t \sim 20$.

In Figs. 3 and 4 we show for $\theta=90^{\circ}$ the effect of taking into account the $C^{1}$ and $C^{2}$ correction factors for longitudinal $\mathrm{W}$ bosons and left- and right-handed electrons, respectively. To visualize these effects we have chosen the extreme mass values $M_{\mathrm{H}}=1000 \mathrm{GeV}$ and $m_{\mathrm{t}}=250 \mathrm{GeV}$. For fermionic and bosonic as well as left- and right-handed electrons we observe a definite improvement of the approximation by consecutively switching on the additional leading $\left(C^{1}\right)$ and subleading $\left(C^{2}\right)$ Higgs- and top-mass-dependent terms. This tendency extends over the full angular range under investigation. The spikes at $280 \mathrm{GeV}$ for the fermionic corrections and at $1091 \mathrm{GeV}$ for the bosonic corrections arise from the thresholds at $\sqrt{s}=2 m_{\mathrm{t}}$ and $\sqrt{s}=M_{\mathrm{Z}}+M_{\mathrm{H}}$, respectively.

The effect of exactly taking into account the leading low-energy corrections, indicated by $\Delta \alpha, \Delta \rho$ and $\Delta_{\mathrm{LL}}$, is displayed in Fig. 5 for the three non-suppressed polarizations and $90^{\circ}$ scattering angle. For the fermionic correction factors this procedure indeed improves the approximations considerably. For intermediate energies the approximations get better by a factor of roughly 4 for longitudinal $\mathrm{W}$ bosons and 10 for transverse $\mathrm{W}$ bosons. On the other hand we find in general no improvement for the bosonic corrections. This can be explained as follows. While the leading low-energy corrections dominate the fermionic corrections, this is not the case for the bosonic corrections. Here the other high-energy leading logarithms, in particular the terms containing $L_{s}^{2}$ and $L_{s}\left(L_{s}-L_{t}\right)$, are comparable with the contributions of $\Delta_{\mathrm{LL}}$ or even exceed them. As a consequence one would have to consider in addition the exact form of these terms so as to obtain a real improvement also for the bosonic corrections. Unfortunately the exact coefficients of the leading high-energy logarithms are not known. Therefore we will only include the fermionic improvement $(\Delta \alpha$, $\Delta \rho)$ and omit the bosonic improvement $\left(\Delta_{\mathrm{LL}}\right)$ in the following, as has already been done in Figs. 3 and 4. We would like to note, however, that the exact treatment of $\Delta_{\text {LL }}$ yields a definite improvement whenever $\Delta_{\mathrm{LL}}$ is dominant. This is in particular the case for small energies $(<500 \mathrm{GeV})$ in the forward direction, where the deviation for the unpolarized and transverse cross-section is below $\sim 1 \%$ if the bosonic improvement is made. The latter pays off also if a small soft-photon cut-off is used such that the terms $\log \left(s / m_{\mathrm{e}}^{2}\right) \log (\Delta E / E)$ become dominant.

The energy dependence of the full approximations is given in Fig. 6. The dips at $280 \mathrm{GeV}$ arise from the top-quark threshold and are not to be confused with cross-overs of the approximation and the exact result. The quality of the approximation improves as expected with energy. The angular dependence for $\sqrt{s}=500 \mathrm{GeV}, 1 \mathrm{TeV}, 2 \mathrm{TeV}$ and $10 \mathrm{TeV}$ is studied in Fig. 7. For energies above $500 \mathrm{GeV}$ the deviation of the approximation 
from the exact result is below $1 \%$ for right-handed electrons at almost all scattering angles, for $(-, L)$ at scattering angles around $90^{\circ}$ (central region), and for $(-, T)$ in the forward hemisphere. This is more or less in agreement with the standards set by the lowest-order approximations, bearing in mind that the corrections can become sizeable at high energies (see Table 1 ). The deviation is larger than $1 \%$ for $(-, T)$ below $\sim 1 \mathrm{TeV}$ in the backward direction and for $(-, L)$ below $2-3 \mathrm{TeV}$ mainly in the forward direction. This is probably due to suppressed terms containing $M_{\mathrm{W}}^{2} / t$ or $M_{\mathrm{W}}^{2} / u$, present in the exact corrections. The effects of such terms can be seen by comparing the approximations for $\theta=90^{\circ}$ with those of $\theta=25^{\circ}$ or $\theta=155^{\circ}$. Note that the approximation is in general best where the cross-sections are maximal, i.e. in the forward direction for transverse $\mathrm{W}$ bosons and in the central region for longitudinal $\mathrm{W}$ bosons. This is probably due to the fact that the relative corrections are comparably small in these regions.

Using the exact lowest-order cross-section as normalization, we show in Figs. 8 and 9 the quality of the approximation for the unpolarized cross-section (20) with the $\Delta \alpha$ and $\Delta \rho$ contributions improved as described in Section 4 also for the suppressed polarizations. Immediately the analogy with the plots for transverse $W$ bosons catches the eye, especially for high energies. Accordingly the deviation of the approximation is of the order of $1 \%$ for $500 \mathrm{GeV}$ and less than $1 \%$ for $1 \mathrm{TeV}$, except in the backward direction, where the crosssection is small compared with the one in the forward direction and where less accuracy is therefore required.

Finally we present in Fig. 10 the quality of the approximations for the various polarized cross-sections integrated over $-0.9 \leq \cos \theta \leq 0.9$. For the unpolarized cross-section, the dominant polarization $(-, T)$, and the polarization $(+, L)$, the error is below $1 \%$ above $500 \mathrm{GeV}$ whereas for the polarization $(-, L)$ this is the case above $700 \mathrm{GeV}$.

\section{Conclusion}

$\mathrm{W}$-pair production is a very important process for investigating the non-Abelian triple gauge couplings. The potential to study these couplings in general increases with energy. The complete $\mathcal{O}(\alpha)$ corrections for the on-shell W-pair production cross-section are very complicated and lengthy. In view of the relevance of these corrections at high energies we have constructed a high-energy approximation.

This approximation is based on the assumption that $s,|t|,|u| \gg M_{\mathrm{W}}^{2}$, whereas the top and Higgs masses can be arbitrary. In addition we have evaluated our general approximation for four interesting special cases. For $M_{\mathrm{W}}^{2} \ll s \ll M_{\mathrm{H}}^{2}$ and $M_{\mathrm{W}}^{2} \ll s \ll m_{\mathrm{t}}^{2}$ we observed large delayed unitarity effects, verified the screening theorem, and recovered the low-energy leading corrections. In the limits $M_{\mathrm{W}}^{2} \ll M_{\mathrm{H}}^{2} \ll s$ and $M_{\mathrm{W}}^{2} \ll m_{\mathrm{t}}^{2} \ll s$ we found for longitudinal gauge bosons additional contributions proportional to $M_{\mathrm{H}}^{2} / M_{\mathrm{W}}^{2}$ and $m_{\mathrm{t}}^{2} / M_{\mathrm{W}}^{2}$, respectively, related to the unitarity cancellations.

We have improved our approximation for intermediate energies by treating the leading low-energy universal corrections associated with the running of $\alpha$ and the $\rho$-parameter exactly. Applying the same procedure to the leading virtual and soft QED logarithms proved to be inappropriate for our purposes.

Where the cross-sections are sizeable, the final approximation matches the required accuracy of roughly $1 \%$ at $500 \mathrm{GeV}$. Over the angular range $-0.9 \leq \cos \theta \leq 0.9$ the deviation from the full virtual and soft-photonic $\mathcal{O}(\alpha)$ corrections is smaller than the expected 
statistical error of a $500 \mathrm{GeV} \mathrm{e}^{+} \mathrm{e}^{-}$collider, and below $1 \%$ above $1-3 \mathrm{TeV}$ depending on the polarizations. As expected, the approximations prove to be very good at high energies.

\section{A High-energy approximations for scalar integrals}

For completeness we give a list of all scalar integrals that have been used for the construction of the high-energy approximation. We define the scalar integrals as follows:

$$
\begin{aligned}
& B_{0}\left(p_{1}^{2}, m_{0}, m_{1}\right)=\frac{(2 \pi \mu)^{4-D}}{i \pi^{2}} \int d^{D} q \frac{1}{\left[q^{2}-m_{0}^{2}+i \varepsilon\right]\left[\left(q+p_{1}\right)^{2}-m_{1}^{2}+i \varepsilon\right]}, \\
& C_{0}\left(p_{1}^{2},\left(p_{2}-p_{1}\right)^{2}, p_{2}^{2}, m_{0}, m_{1}, m_{2}\right)=\frac{1}{i \pi^{2}} \int d^{4} q \\
& \quad \times \frac{1}{\left[q^{2}-m_{0}^{2}+i \varepsilon\right]\left[\left(q+p_{1}\right)^{2}-m_{1}^{2}+i \varepsilon\right]\left[\left(q+p_{2}\right)^{2}-m_{2}^{2}+i \varepsilon\right]}, \\
& D_{0}\left(p_{1}^{2},\left(p_{2}-p_{1}\right)^{2},\left(p_{3}-p_{2}\right)^{2}, p_{3}^{2}, p_{2}^{2},\left(p_{3}-p_{1}\right)^{2}, m_{0}, m_{1}, m_{2}, m_{3}\right)=\frac{1}{i \pi^{2}} \int d^{4} q \\
& \quad \times \frac{1}{\left[q^{2}-m_{0}^{2}+i \varepsilon\right]\left[\left(q+p_{1}\right)^{2}-m_{1}^{2}+i \varepsilon\right]\left[\left(q+p_{2}\right)^{2}-m_{2}^{2}+i \varepsilon\right]\left[\left(q+p_{3}\right)^{2}-m_{3}^{2}+i \varepsilon\right]} .
\end{aligned}
$$

In addition to (13) we introduce a few more abbreviations:

$$
\begin{aligned}
& \Delta_{\mathrm{W}}=\frac{2}{4-D}-\gamma_{E}+\log 4 \pi-\log \left(\frac{M_{\mathrm{W}}^{2}}{\mu^{2}}\right), \quad \zeta(2)=\frac{\pi^{2}}{6}, \\
& L_{\mathrm{top}}=\log \left|1-\frac{M_{\mathrm{W}}^{2}}{m_{\mathrm{t}}^{2}}\right|, \quad L_{\mathrm{e}}=\log \left(\frac{m_{\mathrm{e}}^{2}}{M_{\mathrm{W}}^{2}}\right), \quad L_{\gamma}=\log \left(\frac{\lambda^{2}}{M_{\mathrm{W}}^{2}}\right) \text {, } \\
& \beta_{\mathrm{P}}=\sqrt{1-4 \frac{M_{\mathrm{W}}^{2}}{M_{\mathrm{P}}^{2}-i \varepsilon}}, \quad x_{\mathrm{P}}=\frac{1-\beta_{\mathrm{P}}}{1+\beta_{\mathrm{P}}}, \quad L_{x_{\mathrm{P}}}=\log x_{\mathrm{P}}, \\
& \mathrm{P}=\mathrm{Z}, \mathrm{H}, \\
& \beta_{\mathrm{HZ}}=\sqrt{1-4 \frac{M_{\mathrm{Z}}^{2}}{M_{\mathrm{H}}^{2}-i \varepsilon}}, \quad x_{\mathrm{HZ}}=\frac{1-\beta_{\mathrm{HZ}}}{1+\beta_{\mathrm{HZ}}}, \quad L_{x_{\mathrm{HZ}}}=\log x_{\mathrm{HZ}}, \\
& \beta_{\mathrm{Ztop}}=\sqrt{1-4 \frac{m_{\mathrm{t}}^{2}}{M_{\mathrm{Z}}^{2}-i \varepsilon}}, \quad x_{\mathrm{Ztop}}=\frac{1-\beta_{\mathrm{Ztop}}}{1+\beta_{\mathrm{Ztop}}}, \quad L_{x_{\mathrm{Ztop}}}=\log x_{\mathrm{Ztop}} \\
& \beta_{s \mathrm{HZ}}=\sqrt{1-\frac{4 M_{\mathrm{H}} M_{\mathrm{Z}}}{s-\left(M_{\mathrm{H}}-M_{\mathrm{Z}}\right)^{2}+i \varepsilon}}, \quad x_{s \mathrm{HZ}}=\frac{1-\beta_{s \mathrm{HZ}}}{1+\beta_{s \mathrm{HZ}}}, \quad L_{x_{s \mathrm{HZ}}}=\log x_{s \mathrm{HZ}}, \\
& \beta_{s t o p}=\sqrt{1-4 \frac{m_{\mathrm{t}}^{2}}{s+i \varepsilon}}, \quad x_{\text {stop }}=\frac{1-\beta_{\text {stop }}}{1+\beta_{\text {stop }}}, \quad L_{x_{\text {stop }}}=\log x_{\text {stop }} \text {. }
\end{aligned}
$$

We classify the scalar integrals by the various asymptotic limits described above and start with the list of 2-point functions $B_{0}$ and the corresponding derivatives $B_{0}^{\prime}=\partial B_{0} / \partial p_{1}^{2}$, 
which do not simplify in the high-energy limit and have to be taken exactly (here P denotes any massive particle and $L_{\mathrm{W}}=0$ ):

$$
\begin{aligned}
& B_{0}\left(0,0, M_{\mathrm{P}}\right)=\Delta_{\mathrm{W}}+1-L_{\mathrm{P}}, \\
& B_{0}\left(0, M_{\mathrm{P}}, M_{\mathrm{P}}\right)=\Delta_{\mathrm{W}}-L_{\mathrm{P}}, \\
& B_{0}\left(0, M_{\mathrm{P}_{1}}, M_{\mathrm{P}_{2}}\right)=\Delta_{\mathrm{W}}+1-\frac{M_{\mathrm{P}_{1}}^{2}}{M_{\mathrm{P}_{1}}^{2}-M_{\mathrm{P}_{2}}^{2}} L_{\mathrm{P}_{1}}-\frac{M_{\mathrm{P}_{2}}^{2}}{M_{\mathrm{P}_{2}}^{2}-M_{\mathrm{P}_{1}}^{2}} L_{\mathrm{P}_{2}}, \\
& B_{0}\left(M_{\mathrm{P}}^{2}, 0, M_{\mathrm{P}}\right)=\Delta_{\mathrm{W}}+2-L_{\mathrm{P}}, \\
& B_{0}\left(M_{\mathrm{W}}^{2}, M_{\mathrm{P}}, M_{\mathrm{W}}\right)=\Delta_{\mathrm{W}}+2-\frac{M_{\mathrm{P}}^{2}}{2 M_{\mathrm{W}}^{2}}\left(L_{\mathrm{P}}+\beta_{\mathrm{P}} L_{x_{\mathrm{P}}}\right), \\
& B_{0}\left(M_{\mathrm{Z}}^{2}, M_{\mathrm{H}}, M_{\mathrm{Z}}\right)=\Delta_{\mathrm{W}}+2-L_{\mathrm{Z}}-\frac{M_{\mathrm{H}}^{2}}{2 M_{\mathrm{Z}}^{2}}\left(L_{\mathrm{H}}-L_{\mathrm{Z}}+\beta_{\mathrm{HZ}} L_{x_{\mathrm{HZ}}}\right), \\
& B_{0}^{*}\left(M_{\mathrm{Z}}^{2}, m_{\mathrm{t}}, m_{\mathrm{t}}\right)=\Delta_{\mathrm{W}}+2-L_{\mathrm{top}}+\beta_{\mathrm{Ztop}}\left(L_{x_{\mathrm{Ztop}}}-i \pi\right), \\
& B_{0}^{*}\left(M_{\mathrm{P}}^{2}, M_{\mathrm{W}}, M_{\mathrm{W}}\right)=\Delta_{\mathrm{W}}+2+\beta_{\mathrm{P}}\left(L_{x_{\mathrm{P}}}-i \pi\right) \\
& B_{0}(r, 0,0)=\Delta_{\mathrm{W}}+2-\log \left(\frac{-r-i \varepsilon}{M_{\mathrm{W}}^{2}}\right), \quad r \text { real, } \\
& B_{0}\left(r, M_{\mathrm{P}}, 0\right)=\Delta_{\mathrm{W}}+2-L_{\mathrm{P}}+\left(\frac{M_{\mathrm{P}}^{2}}{r}-1\right) \log \left(1-\frac{r+i \varepsilon}{M_{\mathrm{P}}^{2}}\right), \quad r \text { real, } \\
& B_{0}^{\prime}\left(0,0, M_{\mathrm{P}}\right)=\frac{1}{2 M_{\mathrm{P}}^{2}}, \\
& B_{0}^{\prime}\left(0, M_{\mathrm{P}}, M_{\mathrm{P}}\right)=\frac{1}{6 M_{\mathrm{P}}^{2}}, \\
& B_{0}^{\prime}\left(M_{\mathrm{P}}^{2}, 0,0\right)=-\frac{1}{M_{\mathrm{P}}^{2}}, \\
& B_{0}^{\prime}\left(M_{\mathrm{P}}^{2}, \lambda, M_{\mathrm{P}}\right)=-\frac{1}{M_{\mathrm{P}}^{2}}\left[1+\frac{1}{2}\left(L_{\gamma}-L_{\mathrm{P}}\right)\right], \\
& B_{0}^{\prime}\left(M_{\mathrm{W}}^{2}, M_{\mathrm{P}}, M_{\mathrm{W}}\right)=-\frac{1}{2 M_{\mathrm{W}}^{2}}\left[2+\frac{M_{\mathrm{W}}^{2}-M_{\mathrm{P}}^{2}}{M_{\mathrm{W}}^{2}} L_{\mathrm{P}}+\frac{3 M_{\mathrm{W}}^{2}-M_{\mathrm{P}}^{2}}{M_{\mathrm{W}}^{2} \beta_{\mathrm{P}}} L_{x_{\mathrm{P}}}\right] \text {, } \\
& B_{0}^{\prime}\left(M_{\mathrm{W}}^{2}, 0, m_{\mathrm{t}}\right)=-\frac{1}{M_{\mathrm{W}}^{2}}\left[1+\frac{m_{\mathrm{t}}^{2}}{M_{\mathrm{W}}^{2}} \log \left(1-\frac{M_{\mathrm{W}}^{2}+i \varepsilon}{m_{\mathrm{t}}^{2}}\right)\right] .
\end{aligned}
$$

For the limit $s,-t,-u \gg M_{\mathrm{P}}^{2}$ (with $M_{\mathrm{P}}$ denoting arbitrary masses) all scalar integrals appearing in the full $\mathcal{O}(\alpha)$ expressions have to be expanded accordingly:

$$
\begin{gathered}
B_{0}\left(r, M_{\mathrm{P}_{1}}, M_{\mathrm{P}_{2}}\right) \sim B^{r}=B_{0}(r, 0,0), \quad r=s, t, u, \\
C_{0}\left(m_{\mathrm{e}}^{2}, m_{\mathrm{e}}^{2}, s, m_{\mathrm{e}}, \lambda, m_{\mathrm{e}}\right) \sim \frac{1}{s}\left[\frac{1}{2}\left(L_{\mathrm{e}}-L_{s}+i \pi\right)\left(2 L_{\gamma}-L_{\mathrm{e}}-L_{s}+i \pi\right)-\zeta(2)\right],
\end{gathered}
$$




$$
\begin{aligned}
& C_{0}\left(m_{\mathrm{e}}^{2}, m_{\mathrm{e}}^{2}, s, 0, m_{\mathrm{e}}, M_{\mathrm{Z}}\right) \sim \frac{1}{s}\left[\frac{1}{2}\left(L_{s}-L_{\mathrm{Z}}-i \pi\right)\left(L_{s}+L_{\mathrm{Z}}-2 L_{\mathrm{e}}-i \pi\right)+\zeta(2)\right], \\
& C_{0}\left(0,0, s, 0, M_{\mathrm{P}}, 0\right) \sim \frac{1}{s}\left[\frac{1}{2}\left(L_{s}-L_{\mathrm{P}}-i \pi\right)^{2}+2 \zeta(2)\right], \quad \mathrm{P}=\mathrm{W}, \mathrm{Z}, \\
& C_{0}\left(0,0, s, M_{\mathrm{P}}, 0, M_{\mathrm{P}}\right) \sim \frac{1}{s}\left[\left(L_{s}-L_{\mathrm{P}}-i \pi\right)^{2}\right], \quad \mathrm{P}=\mathrm{W}, \mathrm{Z}, \\
& C_{0}\left(m_{\mathrm{e}}^{2}, M_{\mathrm{W}}^{2}, t, 0, m_{\mathrm{e}}, 0\right) \sim \frac{1}{t}\left[-L_{\mathrm{e}}\left(L_{t}+i \pi\right)+\frac{1}{2} L_{t}^{2}+5 \zeta(2)\right], \\
& C_{0}\left(m_{\mathrm{e}}^{2}, M_{\mathrm{W}}^{2}, r, m_{\mathrm{e}}, \lambda, M_{\mathrm{W}}\right) \sim \frac{1}{r}\left[\frac{1}{2} L_{\gamma}\left(L_{\mathrm{e}}-2 L_{r}\right)-\frac{1}{4} L_{\mathrm{e}}^{2}+\frac{1}{2} L_{r}^{2}-\zeta(2)\right], r=t, u, \\
& C_{0}\left(0, M_{\mathrm{W}}^{2}, t, 0, M_{\mathrm{W}}, 0\right) \sim \frac{1}{t}\left[\frac{1}{2} L_{t}^{2}+3 \zeta(2)\right], \\
& C_{0}\left(0, M_{\mathrm{W}}^{2}, t, M_{\mathrm{Z}}, 0,0\right) \sim \frac{1}{t}\left[\left(L_{t}+i \pi\right)\left(L_{t}-L_{\mathrm{Z}}\right)+\zeta(2)\right], \\
& C_{0}\left(0, M_{\mathrm{W}}^{2}, t, 0, M_{\mathrm{W}}, M_{\mathrm{P}}\right) \sim C_{\nu \mathrm{WP}}^{s}=\frac{1}{t}\left[\frac{1}{2} L_{t}^{2}-\frac{1}{2} L_{x_{\mathrm{P}}}^{2}\right], \quad \mathrm{P}=\mathrm{Z}, \mathrm{H}, \\
& C_{0}\left(0, M_{\mathrm{W}}^{2}, r, 0, M_{\mathrm{Z}}, M_{\mathrm{W}}\right) \sim \frac{1}{r}\left[\frac{1}{2}\left(L_{\mathrm{Z}}-L_{r}\right)^{2}+\frac{1}{4} L_{x_{\mathrm{Z}}}^{2}-\frac{1}{4} L_{\mathrm{Z}}^{2}+2 \zeta(2)\right], r=t, u, \\
& C_{0}\left(0, M_{\mathrm{W}}^{2}, u, M_{\mathrm{W}}, 0,0\right) \sim \frac{1}{u}\left[L_{u}\left(L_{u}+i \pi\right)+\zeta(2)\right], \\
& C_{0}\left(M_{\mathrm{W}}^{2}, M_{\mathrm{W}}^{2}, s, 0,0,0\right) \sim \frac{1}{s}\left[L_{s}^{2}+2 \zeta(2)\right], \\
& C_{0}\left(M_{\mathrm{W}}^{2}, M_{\mathrm{W}}^{2}, s, 0, M_{\mathrm{W}}, M_{\mathrm{Z}}\right) \sim \frac{1}{s}\left[\frac{1}{2}\left(L_{s}-i \pi\right)^{2}-\frac{1}{2} L_{x_{\mathrm{Z}}}^{2}+\zeta(2)\right], \\
& C_{0}\left(M_{\mathrm{W}}^{2}, M_{\mathrm{W}}^{2}, s, M_{\mathrm{W}}, \lambda, M_{\mathrm{W}}\right) \sim \frac{1}{s}\left[-L_{\gamma}\left(L_{s}-i \pi\right)+\frac{1}{2}\left(L_{s}-i \pi\right)^{2}-\zeta(2)\right], \\
& \begin{aligned}
C_{0}\left(M_{\mathrm{P}}^{2}, M_{\mathrm{P}}^{2}, s, 0, M_{\mathrm{P}}, 0\right) & \sim \frac{1}{s}\left[\frac{1}{2}\left(L_{s}-L_{\mathrm{P}}-i \pi\right)^{2}+4 \zeta(2)\right], \\
C_{0}\left(M_{\mathrm{W}}^{2}, M_{\mathrm{W}}^{2}, s, M_{\mathrm{Z}}, M_{\mathrm{W}}, M_{\mathrm{Z}}\right) & \sim \frac{1}{s}\left[\frac{1}{2}\left(L_{s}-i \pi\right)^{2}-L_{x_{\mathrm{Z}}}^{2}-2 \zeta(2)\right],
\end{aligned} \\
& C_{0}\left(M_{\mathrm{W}}^{2}, M_{\mathrm{W}}^{2}, s, M_{\mathrm{W}}, M_{\mathrm{P}}, M_{\mathrm{W}}\right) \sim C_{\mathrm{WPW}}^{s}=\frac{1}{s}\left[\frac{1}{2}\left(L_{s}-i \pi\right)\left(L_{s}-2 L_{\mathrm{P}}-i \pi\right)+\frac{1}{2} L_{x_{\mathrm{P}}}^{2}\right. \\
& +2 \zeta(2)], \quad \mathrm{P}=\mathrm{Z}, \mathrm{H}, \\
& C_{0}\left(M_{\mathrm{W}}^{2}, M_{\mathrm{W}}^{2}, s, M_{\mathrm{H}}, M_{\mathrm{W}}, M_{\mathrm{Z}}\right) \sim C_{\mathrm{HWZ}}^{s}=\frac{1}{s}\left[\frac{1}{2}\left(L_{s}-i \pi\right)^{2}-\frac{1}{2} L_{x_{\mathrm{Z}}}^{2}-\frac{1}{2} L_{x_{\mathrm{H}}}^{2}-2 \zeta(2)\right] \text {, } \\
& C_{0}\left(M_{\mathrm{W}}^{2}, M_{\mathrm{W}}^{2}, s, 0, m_{\mathrm{t}}, 0\right) \sim \frac{1}{s}\left[2 \mathrm{Li}_{2}\left(\frac{M_{\mathrm{W}}^{2}}{m_{\mathrm{t}}^{2}}+i \varepsilon\right)+\frac{1}{2}\left(L_{s}-L_{\mathrm{top}}-i \pi\right)^{2}+2 \zeta(2)\right], \\
& C_{0}\left(M_{\mathrm{W}}^{2}, M_{\mathrm{W}}^{2}, s, m_{\mathrm{t}}, 0, m_{\mathrm{t}}\right) \sim \frac{1}{s}\left[-2 \mathrm{Li}_{2}\left(\frac{M_{\mathrm{W}}^{2}}{m_{\mathrm{t}}^{2}}+i \varepsilon\right)+\left(L_{s}-L_{\mathrm{top}}-i \pi\right)^{2}\right. \\
& \left.-2\left(L_{s}-L_{\mathrm{top}}-i \pi\right) \log \left(1-\frac{M_{\mathrm{W}}^{2}+i \varepsilon}{m_{\mathrm{t}}^{2}}\right)\right] \text {, } \\
& \mathrm{P}=\mathrm{W}, \mathrm{e}
\end{aligned}
$$




$$
\begin{gathered}
D_{0}\left(m_{\mathrm{e}}^{2}, m_{\mathrm{e}}^{2}, M_{\mathrm{W}}^{2}, M_{\mathrm{W}}^{2}, s, r, \lambda, m_{\mathrm{e}}, M_{\mathrm{Z}}, M_{\mathrm{W}}\right) \\
\sim-\frac{1}{s r}\left[\left(\frac{1}{2} L_{\gamma}-L_{s}+\frac{1}{2} L_{\mathrm{Z}}+i \pi\right)\left(2 L_{r}-L_{\mathrm{e}}\right)+\frac{1}{4}\left(L_{\mathrm{e}}-L_{\mathrm{Z}}\right)^{2}+\frac{1}{4} L_{x_{\mathrm{Z}}}^{2}+3 \zeta(2)\right], \\
r=t, u,
\end{gathered}
$$

$D_{0}\left(m_{\mathrm{e}}^{2}, m_{\mathrm{e}}^{2}, M_{\mathrm{W}}^{2}, M_{\mathrm{W}}^{2}, s, t, M_{\mathrm{W}}, 0, M_{\mathrm{W}}, \lambda\right)$

$$
\sim \frac{1}{s t}\left[-\frac{1}{2}\left(2 L_{\gamma}-L_{s}-4 L_{t}+i \pi\right)\left(L_{s}-i \pi\right)-\zeta(2)\right],
$$

$D_{0}\left(m_{\mathrm{e}}^{2}, m_{\mathrm{e}}^{2}, M_{\mathrm{W}}^{2}, M_{\mathrm{W}}^{2}, s, t, m_{\mathrm{e}}, \lambda, m_{\mathrm{e}}, 0\right)$

$$
\sim \frac{1}{s t}\left[\frac{1}{2}\left(2 L_{\gamma}-L_{s}-4 L_{t}-L_{\mathrm{e}}-3 i \pi\right)\left(L_{\mathrm{e}}-L_{s}+i \pi\right)-\zeta(2)\right],
$$

$D_{0}\left(m_{\mathrm{e}}^{2}, M_{\mathrm{W}}^{2}, m_{\mathrm{e}}^{2}, M_{\mathrm{W}}^{2}, t, u, 0, M_{\mathrm{W}}, \lambda, m_{\mathrm{e}}\right)$

$$
\sim \frac{1}{u t}\left[\left(L_{\gamma}-\frac{1}{4} L_{\mathrm{e}}-2 L_{t}-\frac{1}{2} L_{u}\right)\left(\frac{1}{2} L_{\mathrm{e}}-L_{u}\right)-\frac{1}{8} L_{\mathrm{e}}^{2}+2 \zeta(2)+i \pi\left(L_{u}-L_{\mathrm{e}}\right)\right],
$$

$D_{0}\left(0,0, M_{\mathrm{W}}^{2}, M_{\mathrm{W}}^{2}, s, t, 0, M_{\mathrm{Z}}, 0,0\right)$

$$
\sim-\frac{1}{s t}\left[\frac{1}{2}\left(L_{s}-L_{\mathrm{Z}}-i \pi\right)^{2}-\left(L_{s}+L_{t}-L_{\mathrm{Z}}\right)^{2}\right]
$$

$D_{0}\left(0,0, M_{\mathrm{W}}^{2}, M_{\mathrm{W}}^{2}, s, u, 0, M_{\mathrm{W}}, 0,0\right)$

$$
\sim-\frac{1}{s u}\left[\frac{1}{2}\left(L_{s}-i \pi\right)^{2}-\left(L_{u}+L_{s}\right)^{2}\right],
$$

$D_{0}\left(0,0, M_{\mathrm{W}}^{2}, M_{\mathrm{W}}^{2}, s, r, M_{\mathrm{Z}}, 0, M_{\mathrm{Z}}, M_{\mathrm{W}}\right)$

$$
\sim \frac{1}{s r}\left[\frac{1}{2}\left(L_{s}-L_{\mathrm{Z}}-i \pi\right)\left(4 L_{r}+L_{s}-3 L_{\mathrm{Z}}-i \pi\right)-\frac{1}{2} L_{x_{\mathrm{Z}}}^{2}-4 \zeta(2)\right], r=t, u,
$$

$D_{0}\left(0,0, M_{\mathrm{W}}^{2}, M_{\mathrm{W}}^{2}, s, t, M_{\mathrm{W}}, 0, M_{\mathrm{W}}, M_{\mathrm{P}}\right) \sim D_{\mathrm{W} \nu \mathrm{WP}}^{s}$

$$
=\frac{1}{s t}\left[\frac{1}{2}\left(L_{s}-i \pi\right)\left(4 L_{t}+L_{s}-2 L_{\mathrm{P}}-i \pi\right)-\frac{1}{2} L_{x_{\mathrm{P}}}^{2}-4 \zeta(2)\right], \quad \mathrm{P}=\mathrm{Z}, \mathrm{H},
$$

$D_{0}\left(0, M_{\mathrm{W}}^{2}, 0, M_{\mathrm{W}}^{2}, t, u, 0, M_{\mathrm{W}}, M_{\mathrm{Z}}, 0\right)$

$$
\sim \frac{1}{u t}\left[\frac{1}{2}\left(L_{t}+L_{u}-L_{\mathrm{Z}}+i \pi\right)^{2}+L_{u} L_{t}-\frac{1}{4} L_{x_{\mathrm{Z}}}^{2}-\frac{1}{4} L_{\mathrm{Z}}^{2}+\zeta(2)\right] .
$$

For the (sub)leading additional terms $C^{1,2}$ we also need those scalar integrals that involve the Higgs or top mass in the limit $s,-t,-u \sim M_{\mathrm{H}}^{2}, m_{\mathrm{t}}^{2} \gg M_{\mathrm{W}}^{2}$. In $B_{0}\left(s, M_{\mathrm{H}}, M_{\mathrm{Z}}\right)$ and $C_{0}\left(M_{\mathrm{W}}^{2}, M_{\mathrm{W}}^{2}, s, M_{\mathrm{H}}, M_{\mathrm{W}}, M_{\mathrm{Z}}\right)$ we keep the Z-boson mass exactly where necessary in order to describe the threshold at $s=\left(M_{\mathrm{H}}+M_{\mathrm{Z}}\right)^{2}$ and the large $M_{\mathrm{H}}$ limit properly:

$$
\begin{aligned}
& B_{0}\left(s, M_{\mathrm{H}}, M_{\mathrm{Z}}\right)=B_{\mathrm{HZ}}= \Delta_{\mathrm{W}}+2-\frac{s+M_{\mathrm{H}}^{2}-M_{\mathrm{Z}}^{2}}{2 s} L_{\mathrm{H}}-\frac{s+M_{\mathrm{Z}}^{2}-M_{\mathrm{H}}^{2}}{2 s} L_{\mathrm{Z}} \\
&+\frac{s-\left(M_{\mathrm{H}}-M_{\mathrm{Z}}\right)^{2}}{s} \beta_{s \mathrm{HZ}}\left(L_{x_{s \mathrm{HZ}}}+i \pi\right), \\
& B_{0}\left(s, m_{\mathrm{t}}, m_{\mathrm{t}}\right)=B_{\mathrm{tt}}= \Delta_{\mathrm{W}}+2-L_{\mathrm{top}}+\beta_{s \mathrm{top}}\left(L_{x_{s \mathrm{op}}}+i \pi\right), \\
& C_{0}\left(0, M_{\mathrm{W}}^{2}, t, 0, M_{\mathrm{W}}, M_{\mathrm{H}}\right) \sim C_{\nu \mathrm{WH}}^{h}=\frac{1}{t}\left[\frac{1}{2} \log ^{2}\left(\frac{M_{\mathrm{H}}^{2}-t}{M_{\mathrm{H}}^{2}}\right)+L_{\mathrm{H}} \log \left(\frac{M_{\mathrm{H}}^{2}-t}{\left.\left.M_{\mathrm{H}}^{2}\right)\right],}\right.\right. \\
& C_{0}\left(M_{\mathrm{W}}^{2}, M_{\mathrm{W}}^{2}, s, M_{\mathrm{W}}, M_{\mathrm{H}}, M_{\mathrm{W}}\right) \sim C_{\mathrm{WHW}}^{h}=\frac{1}{s}\left[-\operatorname{Li}_{2}\left(1+\frac{s}{M_{\mathrm{H}}^{2}}+i \varepsilon\right)+\zeta(2)\right]
\end{aligned}
$$




$$
\begin{gathered}
C_{0}\left(M_{\mathrm{W}}^{2}, M_{\mathrm{W}}^{2}, s, M_{\mathrm{H}}, M_{\mathrm{W}}, M_{\mathrm{Z}}\right) \sim C_{\mathrm{HWZ}}^{h}=\frac{1}{s}\left[\mathrm{Li}_{2}\left(\frac{s+i \varepsilon}{M_{\mathrm{H}} M_{\mathrm{Z}}} x_{s \mathrm{HZ}}\right)-\frac{1}{2} L_{\mathrm{H}}^{2}-\frac{1}{2} L_{x_{\mathrm{Z}}}^{2}\right. \\
+\frac{1}{2} \log ^{2}\left(-\frac{M_{\mathrm{W}}^{2}}{M_{\mathrm{H}} M_{\mathrm{Z}}} x_{s \mathrm{HZ}}\right)-3 \zeta(2)+\sum_{\rho= \pm 1}\left\{\operatorname{Li}_{2}\left(-\frac{M_{\mathrm{H}}}{M_{\mathrm{Z}}} x_{s \mathrm{HZ}}\left(1+x_{\mathrm{Z}}^{\rho}\right)\right)\right. \\
\left.\left.\quad+\log \left(1+\frac{M_{\mathrm{H}}}{M_{\mathrm{Z}}} x_{s \mathrm{HZ}}\left(1+x_{\mathrm{Z}}^{\rho}\right)\right)\left(\log \left(-\frac{M_{\mathrm{H}}}{M_{\mathrm{Z}}} x_{s \mathrm{HZ}}\right)+\log \left(1+x_{\mathrm{Z}}^{\rho}\right)\right)\right\}\right] \\
C_{0}\left(M_{\mathrm{W}}^{2}, M_{\mathrm{W}}^{2}, s, 0, m_{\mathrm{t}}, 0\right) \sim C_{\mathrm{btb}}^{h}=\frac{1}{s}\left[-\mathrm{Li}_{2}\left(1+\frac{s}{m_{\mathrm{t}}^{2}}+i \varepsilon\right)+\zeta(2)\right] \\
C_{0}\left(M_{\mathrm{W}}^{2}, M_{\mathrm{W}}^{2}, s, m_{\mathrm{t}}, 0, m_{\mathrm{t}}\right) \sim C_{\mathrm{tbt}}^{h}=\frac{1}{s}\left(L_{x_{s \mathrm{top}}}+i \pi\right)^{2}, \\
D_{0}\left(0,0, M_{\mathrm{W}}^{2}, M_{\mathrm{W}}^{2}, s, t, M_{\mathrm{W}}, 0, M_{\mathrm{W}}, M_{\mathrm{H}}\right) \sim D_{\mathrm{W} \nu \mathrm{WH}}^{h}=\frac{1}{s\left(t-M_{\mathrm{H}}^{2}\right)}\left[2 \mathrm{Li}_{2}\left(\frac{t}{M_{\mathrm{H}}^{2}}\right)\right. \\
\left.+\mathrm{Li}_{2}\left(1+\frac{s}{M_{\mathrm{H}}^{2}}+i \varepsilon\right)+\left(\log \left(\frac{M_{\mathrm{H}}^{2}-t}{M_{\mathrm{H}}^{2}}\right)+L_{s}-i \pi\right)^{2}-\zeta(2)\right]
\end{gathered}
$$

Finally all scalar integrals entering the calculation of $C^{1}$ have to be expanded up to order $M_{\mathrm{W}}^{2} / s$ to obtain the correct subleading corrections $C^{2}$ :

$$
\begin{aligned}
B_{0}\left(s, M_{\mathrm{W}}, M_{\mathrm{W}}\right) \sim \Delta_{\mathrm{W}}+2 & -L_{s}+i \pi+\frac{2 M_{\mathrm{W}}^{2}}{s}\left(1+L_{s}-i \pi\right), \\
C_{0}\left(M_{\mathrm{W}}^{2}, M_{\mathrm{W}}^{2}, s, M_{\mathrm{W}}, M_{\mathrm{H}}, M_{\mathrm{W}}\right) \sim C_{\mathrm{WHW}}^{h 1}= & C_{\mathrm{WHW}}^{h}\left(1+\frac{2 M_{\mathrm{W}}^{2}}{s}\right) \\
& +\frac{4 M_{\mathrm{W}}^{2}}{s}\left(\frac{L_{\mathrm{H}}-L_{s}+i \pi}{s+M_{\mathrm{H}}^{2}}-\frac{L_{\mathrm{H}}}{2 M_{\mathrm{H}}^{2}}\right), \\
C_{0}\left(M_{\mathrm{W}}^{2}, M_{\mathrm{W}}^{2}, s, 0, m_{\mathrm{t}}, 0\right) \sim C_{\mathrm{btb}}^{h 1}=C_{\mathrm{btb}}^{h}\left(1+\frac{2 M_{\mathrm{W}}^{2}}{s}\right) & +\frac{2 M_{\mathrm{W}}^{2}}{s}\left(\frac{1}{m_{\mathrm{t}}^{2}}-\frac{L_{s}-L_{\mathrm{top}}-i \pi}{s+m_{\mathrm{t}}^{2}}\right), \\
C_{0}\left(M_{\mathrm{W}}^{2}, M_{\mathrm{W}}^{2}, s, m_{\mathrm{t}}, 0, m_{\mathrm{t}}\right) \sim C_{\mathrm{tbt}}^{h 1}=C_{\mathrm{tbt}}^{h}\left(1+\frac{2 M_{\mathrm{W}}^{2}}{s}\right) & -\frac{2 M_{\mathrm{W}}^{2}}{s m_{\mathrm{t}}^{2}}\left[\beta_{s \mathrm{top}}\left(L_{x_{s \mathrm{top}}}+i \pi\right)+1\right] .
\end{aligned}
$$




\section{B High-energy correction factors}

In this appendix we list the remaining non-leading correction factors of the highenergy approximation discussed in Section 3 as well as the contributions of the mixed polarizations to the cross-section for unpolarized $\mathrm{W}$ bosons:

$$
\begin{aligned}
& C_{-, L}^{B, \text { const }}=\frac{\alpha}{2 \pi}\left[-\frac{\left(1-2 c_{\mathrm{W}}^{2}\right)^{2}}{4 c_{\mathrm{W}}^{2} s_{\mathrm{W}}^{2}} L_{\mathrm{Z}}^{2}-\frac{2+18 c_{\mathrm{W}}^{2}-75 c_{\mathrm{W}}^{4}+26 c_{\mathrm{W}}^{6}+60 c_{\mathrm{W}}^{8}-120 c_{\mathrm{W}}^{10}}{24 c_{\mathrm{W}}^{6} s_{\mathrm{W}}^{4}} L_{\mathrm{Z}}\right. \\
& -\frac{1-6 c_{\mathrm{W}}^{2}+4 c_{\mathrm{W}}^{4}}{4 c_{\mathrm{W}}^{2} s_{\mathrm{W}}^{2}} L_{x_{\mathrm{Z}}}^{2}-\frac{2+22 c_{\mathrm{W}}^{2}-71 c_{\mathrm{W}}^{4}+56 c_{\mathrm{W}}^{6}-108 c_{\mathrm{W}}^{8}}{24 c_{\mathrm{W}}^{6} s_{\mathrm{W}}^{4}} \beta_{\mathrm{Z}} L_{x_{\mathrm{Z}}} \\
& +\frac{\left(1-2 c_{\mathrm{W}}^{2}\right)\left(1-4 c_{\mathrm{W}}^{2}\right)\left(1+20 c_{\mathrm{W}}^{2}+12 c_{\mathrm{W}}^{4}\right)}{12 c_{\mathrm{W}}^{2} s_{\mathrm{W}}^{4}} \beta_{\mathrm{Z}}\left(L_{x_{\mathrm{Z}}}-i \pi\right) \\
& \left.+\frac{4+19 c_{\mathrm{W}}^{2}+42 c_{\mathrm{W}}^{4}-324 c_{\mathrm{W}}^{6}-384 c_{\mathrm{W}}^{8}}{24 c_{\mathrm{W}}^{4} s_{\mathrm{W}}^{2}}+\frac{1+6 c_{\mathrm{W}}^{2}-12 c_{\mathrm{W}}^{4}}{c_{\mathrm{W}}^{2} s_{\mathrm{W}}^{2}} \zeta(2)\right] \\
& C_{+, L}^{B, \text { const }}=\frac{\alpha}{2 \pi}\left[-\frac{s_{\mathrm{W}}^{2}}{c_{\mathrm{W}}^{2}} L_{\mathrm{Z}}^{2}-\frac{2+19 c_{\mathrm{W}}^{2}-16 c_{\mathrm{W}}^{4}-12 c_{\mathrm{W}}^{6}+120 c_{\mathrm{W}}^{8}}{24 c_{\mathrm{W}}^{6} s_{\mathrm{W}}^{2}} L_{\mathrm{Z}}-\frac{1-6 c_{\mathrm{W}}^{2}+4 c_{\mathrm{W}}^{4}}{4 c_{\mathrm{W}}^{2} s_{\mathrm{W}}^{2}} L_{x_{\mathrm{Z}}}^{2}\right. \\
& -\frac{2+23 c_{\mathrm{W}}^{2}-64 c_{\mathrm{W}}^{4}+60 c_{\mathrm{W}}^{6}}{24 c_{\mathrm{W}}^{6} s_{\mathrm{W}}^{2}} \beta_{\mathrm{Z}} L_{x_{\mathrm{Z}}}+\frac{\left(1-4 c_{\mathrm{W}}^{2}\right)\left(1+20 c_{\mathrm{W}}^{2}+12 c_{\mathrm{W}}^{4}\right)}{12 c_{\mathrm{W}}^{2} s_{\mathrm{W}}^{2}} \beta_{\mathrm{Z}}\left(L_{x_{\mathrm{Z}}}-i \pi\right) \\
& \left.+\frac{2-23 c_{\mathrm{W}}^{2}+78 c_{\mathrm{W}}^{4}-122 c_{\mathrm{W}}^{6}-96 c_{\mathrm{W}}^{8}}{12 c_{\mathrm{W}}^{4} s_{\mathrm{W}}^{2}}+\frac{5+6 c_{\mathrm{W}}^{2}}{2 c_{\mathrm{W}}^{2} s_{\mathrm{W}}^{2}} \zeta(2)\right] \\
& C_{-, T}^{B, \text { const }}=\frac{\alpha}{2 \pi}\left[-\frac{\left(1-2 c_{\mathrm{W}}^{2}\right)^{2}}{4 c_{\mathrm{W}}^{2} s_{\mathrm{W}}^{2}} L_{\mathrm{Z}}^{2}-\frac{2+18 c_{\mathrm{W}}^{2}-101 c_{\mathrm{W}}^{4}+52 c_{\mathrm{W}}^{6}+60 c_{\mathrm{W}}^{8}-120 c_{\mathrm{W}}^{10}}{24 c_{\mathrm{W}}^{6} s_{\mathrm{W}}^{4}} L_{\mathrm{Z}}\right. \\
& +\frac{c_{\mathrm{W}}^{2}}{s_{\mathrm{W}}^{2}} L_{x_{\mathrm{Z}}}^{2}-\frac{2+22 c_{\mathrm{W}}^{2}-71 c_{\mathrm{W}}^{4}+56 c_{\mathrm{W}}^{6}-108 c_{\mathrm{W}}^{8}}{24 c_{\mathrm{W}}^{6} s_{\mathrm{W}}^{4}} \beta_{\mathrm{Z}} L_{x_{\mathrm{Z}}} \\
& -\frac{\left(1-4 c_{\mathrm{W}}^{2}\right)\left(1+20 c_{\mathrm{W}}^{2}+12 c_{\mathrm{W}}^{4}\right)}{12 s_{\mathrm{W}}^{4}} \beta_{\mathrm{Z}}\left(L_{x_{\mathrm{Z}}}-i \pi\right) \\
& \left.+\frac{4+25 c_{\mathrm{W}}^{2}+106 c_{\mathrm{W}}^{4}-436 c_{\mathrm{W}}^{6}-192 c_{\mathrm{W}}^{8}}{24 c_{\mathrm{W}}^{4} s_{\mathrm{W}}^{2}}+\frac{1-22 c_{\mathrm{W}}^{2}+24 c_{\mathrm{W}}^{4}}{2 c_{\mathrm{W}}^{2} s_{\mathrm{W}}^{2}} \zeta(2)\right], \\
& C_{-, L}^{F, \text { const }}=\frac{\alpha}{2 \pi}\left[-\frac{\left(1-2 c_{\mathrm{W}}^{2}\right)\left(103-200 c_{\mathrm{W}}^{2}+160 c_{\mathrm{W}}^{4}\right)}{18 c_{\mathrm{W}}^{2} s_{\mathrm{W}}^{4}} L_{\mathrm{Z}}-\frac{4\left(31-40 c_{\mathrm{W}}^{2}\right)}{9 s_{\mathrm{W}}^{2}}\right], \\
& C_{+, L}^{F, \text { const }}=\frac{\alpha}{2 \pi}\left[-\frac{103-200 c_{\mathrm{W}}^{2}+160 c_{\mathrm{W}}^{4}}{18 c_{\mathrm{W}}^{2} s_{\mathrm{W}}^{2}} L_{\mathrm{Z}}-\frac{4\left(31-40 c_{\mathrm{W}}^{2}\right)}{9 s_{\mathrm{W}}^{2}}\right] \text {, } \\
& C_{-, T}^{F, \text { const }}=\frac{\alpha}{2 \pi}\left[\frac{103-200 c_{\mathrm{W}}^{2}+160 c_{\mathrm{W}}^{4}}{18 s_{\mathrm{W}}^{4}} L_{\mathrm{Z}}-\frac{4\left(31-40 c_{\mathrm{W}}^{2}\right)}{9 s_{\mathrm{W}}^{2}}\right], \\
& C_{-, L}^{B, H}=\frac{\alpha}{2 \pi}\left[\frac{1}{2 s_{\mathrm{W}}^{2}}\left(L_{x_{\mathrm{H}}}^{2}-L_{\mathrm{H}}^{2}\right)-\frac{M_{\mathrm{H}}^{2}\left[12 M_{\mathrm{W}}^{4}-\left(5-c_{\mathrm{W}}^{2}\right) M_{\mathrm{W}}^{2} M_{\mathrm{H}}^{2}+\left(2-c_{\mathrm{W}}^{2}\right) M_{\mathrm{H}}^{4}\right]}{24 s_{\mathrm{W}}^{4} M_{\mathrm{W}}^{6}} \beta_{\mathrm{H}} L_{x_{\mathrm{H}}}\right. \\
& -\frac{1}{2 s_{\mathrm{W}}^{2} \beta_{\mathrm{H}}} L_{x_{\mathrm{H}}}-\frac{\left(1-2 c_{\mathrm{W}}^{2}\right)\left(12 M_{\mathrm{Z}}^{4}-4 M_{\mathrm{Z}}^{2} M_{\mathrm{H}}^{2}+M_{\mathrm{H}}^{4}\right) M_{\mathrm{H}}^{2}}{24 s_{\mathrm{W}}^{4} M_{\mathrm{WZ}}^{2} M_{\mathrm{Z}}^{4}} L_{x_{\mathrm{HZ}}} \\
& -\frac{M_{\mathrm{H}}^{2}\left[21 M_{\mathrm{W}}^{2}-4\left(1+c_{\mathrm{W}}^{2}\right) M_{\mathrm{H}}^{2}\right]}{24 s_{\mathrm{W}}^{2} M_{\mathrm{W}}^{4}}+\frac{M_{\mathrm{H}}^{2}\left(1-2 c_{\mathrm{W}}^{2}\right)\left(18 M_{\mathrm{Z}}^{4}-6 M_{\mathrm{Z}}^{2} M_{\mathrm{H}}^{2}+M_{\mathrm{H}}^{4}\right)}{24 s_{\mathrm{W}}^{4} M_{\mathrm{W}}^{2} M_{\mathrm{Z}}^{4}} L_{\mathrm{Z}}
\end{aligned}
$$




$$
\begin{aligned}
& \left.+\frac{12 M_{\mathrm{W}}^{6}-36 M_{\mathrm{W}}^{4} M_{\mathrm{H}}^{2}+3\left(3+4 c_{\mathrm{W}}^{2}\right) M_{\mathrm{W}}^{2} M_{\mathrm{H}}^{4}-\left(2+c_{\mathrm{W}}^{2}+2 c_{\mathrm{W}}^{4}\right) M_{\mathrm{H}}^{6}}{24 s_{\mathrm{W}}^{2} M_{\mathrm{W}}^{6}} L_{\mathrm{H}}\right], \\
& C_{+, L}^{B, H}=\frac{\alpha}{2 \pi}\left[\frac{1}{2 s_{\mathrm{W}}^{2}}\left(L_{x_{\mathrm{H}}}^{2}-L_{\mathrm{H}}^{2}\right)-\frac{M_{\mathrm{H}}^{2}\left(12 M_{\mathrm{W}}^{4}-5 M_{\mathrm{W}}^{2} M_{\mathrm{H}}^{2}+2 M_{\mathrm{H}}^{4}\right)}{24 s_{\mathrm{W}}^{2} M_{\mathrm{W}}^{6}} \beta_{\mathrm{H}} L_{x_{\mathrm{H}}}\right. \\
& -\frac{1}{2 s_{\mathrm{W}}^{2} \beta_{\mathrm{H}}} L_{x_{\mathrm{H}}}-\frac{\left(12 M_{\mathrm{Z}}^{4}-4 M_{\mathrm{Z}}^{2} M_{\mathrm{H}}^{2}+M_{\mathrm{H}}^{4}\right) M_{\mathrm{H}}^{2}}{24 s_{\mathrm{W}}^{2} M_{\mathrm{W}}^{2} M_{\mathrm{Z}}^{4}} \beta_{\mathrm{HZ}} L_{x_{\mathrm{HZ}}} \\
& -\frac{M_{\mathrm{H}}^{2}\left(21 M_{\mathrm{W}}^{2}-2\left(2+c_{\mathrm{W}}^{2}\right) M_{\mathrm{H}}^{2}\right)}{24 s_{\mathrm{W}}^{2} M_{\mathrm{W}}^{4}}+\frac{M_{\mathrm{H}}^{2}\left(18 M_{\mathrm{Z}}^{4}-6 M_{\mathrm{Z}}^{2} M_{\mathrm{H}}^{2}+M_{\mathrm{H}}^{4}\right)}{24 s_{\mathrm{W}}^{2} M_{\mathrm{W}}^{2} M_{\mathrm{Z}}^{4}} L_{\mathrm{Z}} \\
& \left.+\frac{12 M_{\mathrm{W}}^{6}-36 M_{\mathrm{W}}^{4} M_{\mathrm{H}}^{2}+3\left(3+2 c_{\mathrm{W}}^{2}\right) M_{\mathrm{W}}^{2} M_{\mathrm{H}}^{4}-\left(2+c_{\mathrm{W}}^{4}\right) M_{\mathrm{H}}^{6}}{24 s_{\mathrm{W}}^{2} M_{\mathrm{W}}^{6}} L_{\mathrm{H}}\right], \\
& C_{-, T}^{B, H}=\frac{\alpha}{2 \pi}\left[-\frac{M_{\mathrm{H}}^{2}\left[12 M_{\mathrm{W}}^{4}-\left(5-c_{\mathrm{W}}^{2}\right) M_{\mathrm{W}}^{2} M_{\mathrm{H}}^{2}+\left(2-c_{\mathrm{W}}^{2}\right) M_{\mathrm{H}}^{4}\right]}{24 s_{\mathrm{W}}^{4} M_{\mathrm{W}}^{6}} \beta_{\mathrm{H}} L_{x_{\mathrm{H}}}-\frac{1}{2 s_{\mathrm{W}}^{2} \beta_{\mathrm{H}}} L_{x_{\mathrm{H}}}\right. \\
& +\frac{\left(12 M_{\mathrm{Z}}^{4}-4 M_{\mathrm{Z}}^{2} M_{\mathrm{H}}^{2}+M_{\mathrm{H}}^{4}\right) M_{\mathrm{H}}^{2}}{24 s_{\mathrm{W}}^{4} M_{\mathrm{Z}}^{6}} \beta_{\mathrm{HZ}} L_{x_{\mathrm{HZ}}}-\frac{M_{\mathrm{H}}^{2}\left[6 M_{\mathrm{W}}^{2}-\left(2+c_{\mathrm{W}}^{2}\right) M_{\mathrm{H}}^{2}\right]}{12 s_{\mathrm{W}}^{2} M_{\mathrm{W}}^{4}} \\
& -\frac{M_{\mathrm{H}}^{2}\left(18 M_{\mathrm{Z}}^{4}-6 M_{\mathrm{Z}}^{2} M_{\mathrm{H}}^{2}+M_{\mathrm{H}}^{4}\right)}{24 s_{\mathrm{W}}^{4} M_{\mathrm{Z}}^{6}} L_{\mathrm{Z}} \\
& \left.+\frac{12 M_{\mathrm{W}}^{6}-18 M_{\mathrm{W}}^{4} M_{\mathrm{H}}^{2}+3\left(3+2 c_{\mathrm{W}}^{2}\right) M_{\mathrm{W}}^{2} M_{\mathrm{H}}^{4}-\left(2+c_{\mathrm{W}}^{2}+c_{\mathrm{W}}^{4}\right) M_{\mathrm{H}}^{6}}{24 s_{\mathrm{W}}^{2} M_{\mathrm{W}}^{6}} L_{\mathrm{H}}\right] \text {, } \\
& C_{-, L}^{F, t}=\frac{\alpha}{2 \pi}\left[-\frac{17-60 c_{\mathrm{W}}^{2}+84 c_{\mathrm{W}}^{4}-32 c_{\mathrm{W}}^{6}}{18 c_{\mathrm{W}}^{2} s_{\mathrm{W}}^{4}} L_{\mathrm{top}}+\frac{\left(10-367 c_{\mathrm{W}}^{2}+896 c_{\mathrm{W}}^{4}-512 c_{\mathrm{W}}^{6}\right) m_{\mathrm{t}}^{2}}{36 s_{\mathrm{W}}^{4} M_{\mathrm{W}}^{2}}\right. \\
& -\frac{\left(2-c_{\mathrm{W}}^{2}\right) m_{\mathrm{t}}^{4}}{2 s_{\mathrm{W}}^{4} M_{\mathrm{W}}^{4}}+\frac{2\left(1-2 c_{\mathrm{W}}^{2}\right) M_{\mathrm{W}}^{6}+3 c_{\mathrm{W}}^{2} M_{\mathrm{W}}^{4} m_{\mathrm{t}}^{2}-\left(2-c_{\mathrm{W}}^{2}\right) m_{\mathrm{t}}^{6}}{2 s_{\mathrm{W}}^{4} M_{\mathrm{W}}^{6}} L_{\mathrm{top}} \\
& \left.+\left(1-2 c_{\mathrm{W}}^{2}\right) \frac{\left(17-40 c_{\mathrm{W}}^{2}+32 c_{\mathrm{W}}^{4}\right) M_{\mathrm{Z}}^{2}+\left(7-80 c_{\mathrm{W}}^{2}+64 c_{\mathrm{W}}^{4}\right) m_{\mathrm{t}}^{2}}{18 s_{\mathrm{W}}^{4} M_{\mathrm{W}}^{2}} \beta_{\mathrm{Ztop}}\left(L_{x_{\mathrm{Ztop}}}-i \pi\right)\right], \\
& C_{+, L}^{F, t}=\frac{\alpha}{2 \pi}\left[-\frac{17-26 c_{\mathrm{W}}^{2}}{18 c_{\mathrm{W}}^{2} s_{\mathrm{W}}^{2}} L_{\mathrm{top}}+\frac{\left(5-160 c_{\mathrm{W}}^{2}+128 c_{\mathrm{W}}^{4}\right) m_{\mathrm{t}}^{2}}{18 s_{\mathrm{W}}^{2} M_{\mathrm{W}}^{2}}-\frac{m_{\mathrm{t}}^{4}}{s_{\mathrm{W}}^{2} M_{\mathrm{W}}^{4}}+\frac{M_{\mathrm{W}}^{6}-m_{\mathrm{t}}^{6}}{s_{\mathrm{W}}^{2} M_{\mathrm{W}}^{6}} L_{\mathrm{top}}\right. \\
& \left.+\frac{\left(17-40 c_{\mathrm{W}}^{2}+32 c_{\mathrm{W}}^{4}\right) M_{\mathrm{Z}}^{2}+\left(7-80 c_{\mathrm{W}}^{2}+64 c_{\mathrm{W}}^{4}\right) m_{\mathrm{t}}^{2}}{18 s_{\mathrm{W}}^{2} M_{\mathrm{W}}^{2}} \beta_{\mathrm{Ztop}}\left(L_{x_{\mathrm{Ztop}}}-i \pi\right)\right], \\
& C_{-, T}^{F, t}=\frac{\alpha}{2 \pi}\left[\frac{1-4 c_{\mathrm{W}}^{2}}{6 s_{\mathrm{W}}^{4}} L_{\mathrm{top}}-\frac{\left(18+19 c_{\mathrm{W}}^{2}-320 c_{\mathrm{W}}^{4}+256 c_{\mathrm{W}}^{6}\right) m_{\mathrm{t}}^{2}}{36 s_{\mathrm{W}}^{4} M_{\mathrm{W}}^{2}}-\frac{\left(2-c_{\mathrm{W}}^{2}\right) m_{\mathrm{t}}^{4}}{2 s_{\mathrm{W}}^{4} M_{\mathrm{W}}^{4}}\right. \\
& +\frac{2\left(1-2 c_{\mathrm{W}}^{2}\right) M_{\mathrm{W}}^{6}+3 c_{\mathrm{W}}^{2} M_{\mathrm{W}}^{4} m_{\mathrm{t}}^{2}-\left(2-c_{\mathrm{W}}^{2}\right) m_{\mathrm{t}}^{6}}{2 s_{\mathrm{W}}^{4} M_{\mathrm{W}}^{6}} L_{\mathrm{topW}} \\
& \left.-\frac{\left(17-40 c_{\mathrm{W}}^{2}+32 c_{\mathrm{W}}^{4}\right) M_{\mathrm{Z}}^{2}+\left(7-80 c_{\mathrm{W}}^{2}+64 c_{\mathrm{W}}^{4}\right) m_{\mathrm{t}}^{2}}{18 s_{\mathrm{W}}^{4} M_{\mathrm{Z}}^{2}} \beta_{\mathrm{Ztop}}\left(L_{x_{\mathrm{Ztop}}}-i \pi\right)\right] \text {, } \\
& C_{ \pm, L}^{B, 1}=-\frac{\alpha}{2 \pi} \frac{M_{\mathrm{H}}^{4}}{4 s_{\mathrm{W}}^{2} M_{\mathrm{W}}^{2} s}\left[\left(s+2 M_{\mathrm{H}}^{2}\right) C_{\mathrm{WHW}}^{h}+2+2 L_{\mathrm{H}}-2 L_{s}\right], \\
& C_{-, T}^{B, 1}=0,
\end{aligned}
$$




$$
\begin{aligned}
& C_{-, L}^{F, 1}=\frac{\alpha}{2 \pi} \frac{m_{\mathrm{t}}^{2}}{s_{\mathrm{W}}^{2} M_{\mathrm{W}}^{2}}\left[-\left(1-4 c_{\mathrm{W}}^{2}\right) m_{\mathrm{t}}^{2}\left(1+\frac{m_{\mathrm{t}}^{2}}{s}\right) C_{\mathrm{btb}}^{h}-\left(1+2 c_{\mathrm{W}}^{2}\right) \frac{m_{\mathrm{t}}^{4}}{s} C_{\mathrm{tbt}}^{h}\right. \\
& \left.+6 c_{\mathrm{W}}^{2} \frac{m_{\mathrm{t}}^{2}}{s}\left(L_{\mathrm{top}}-L_{s}+1\right)+\left(2 s_{\mathrm{W}}^{2}+\frac{m_{\mathrm{t}}^{2}}{s}\left(1+2 c_{\mathrm{W}}^{2}\right)\right)\left(B_{\mathrm{tt}}-B^{s}\right)\right] \text {, } \\
& C_{+, L}^{F, 1}=\frac{\alpha}{2 \pi} \frac{m_{\mathrm{t}}^{2}}{s_{\mathrm{W}}^{2} M_{\mathrm{W}}^{2}}\left[-m_{\mathrm{t}}^{2}\left(1+\frac{m_{\mathrm{t}}^{2}}{s}\right) C_{\mathrm{btb}}^{h}-\frac{m_{\mathrm{t}}^{4}}{s} C_{\mathrm{tbt}}^{h}+\left(2+\frac{m_{\mathrm{t}}^{2}}{s}\right)\left(B_{\mathrm{tt}}-B^{s}\right)\right], \\
& C_{-, T}^{F, 1}=0, \\
& C_{-, L}^{B, 2}=\frac{\alpha}{2 \pi}\left[\frac{s M_{\mathrm{H}}^{2}\left(23-80 c_{\mathrm{W}}^{2}+32 c_{\mathrm{W}}^{4}\right)-2 M_{\mathrm{H}}^{4}\left(13-4 c_{\mathrm{W}}^{2}+124 c_{\mathrm{W}}^{4}\right)}{24 c_{\mathrm{W}}^{2} s_{\mathrm{W}}^{2} s^{2}}\right. \\
& -\frac{c_{\mathrm{W}}^{2} M_{\mathrm{H}}^{2}\left(4 M_{\mathrm{H}}^{2}+s\right)}{2 s_{\mathrm{W}}^{2} s t}+\frac{M_{\mathrm{H}}^{4}\left(1-2 c_{\mathrm{W}}^{2}\right)}{2 c_{\mathrm{W}}^{2} s_{\mathrm{W}}^{2} s^{2}} L_{\mathrm{Z}}+\frac{M_{\mathrm{H}}^{2}\left(1-2 c_{\mathrm{W}}^{2}\right)\left(M_{\mathrm{H}}^{2}-s\right)}{2 c_{\mathrm{W}}^{2} s_{\mathrm{W}}^{2} s^{2}} \beta_{\mathrm{Z}} L_{x_{\mathrm{Z}}} \\
& -\frac{M_{\mathrm{H}}^{2}\left[s\left(1-4 c_{\mathrm{W}}^{2}+6 c_{\mathrm{W}}^{4}\right)+4 c_{\mathrm{W}}^{2} s_{\mathrm{W}}^{2} M_{\mathrm{H}}^{2}\right]}{2 c_{\mathrm{W}}^{2} s_{\mathrm{W}}^{2} s^{2}} L_{s}-\frac{2 c_{\mathrm{W}}^{2} M_{\mathrm{H}}^{4}}{s_{\mathrm{W}}^{2} s t}\left(L_{\mathrm{H}}-L_{s}\right) \\
& +\frac{M_{\mathrm{H}}^{2}\left[4 s\left(2-8 c_{\mathrm{W}}^{2}+5 c_{\mathrm{W}}^{4}\right)-M_{\mathrm{H}}^{2}\left(13-16 c_{\mathrm{W}}^{2}+124 c_{\mathrm{W}}^{4}\right)\right]}{12 c_{\mathrm{W}}^{2} s_{\mathrm{W}}^{2} s^{2}}\left(L_{\mathrm{H}}-L_{s}\right) \\
& -\frac{\left(1-2 c_{\mathrm{W}}^{2}\right)\left[s^{2}\left(1+22 c_{\mathrm{W}}^{2}\right)-14 s M_{\mathrm{H}}^{2}\left(1-2 c_{\mathrm{W}}^{2}\right)+M_{\mathrm{H}}^{4}\left(1-26 c_{\mathrm{W}}^{2}\right)\right]}{12 c_{\mathrm{W}}^{2} s_{\mathrm{W}}^{2} s^{2}}\left(B_{\mathrm{HZ}}-B^{s}\right) \\
& +\frac{1}{2 s_{\mathrm{W}}^{2}}\left(L_{x_{\mathrm{H}}}^{2}-L_{\mathrm{H}}^{2}\right)-\frac{M_{\mathrm{H}}^{2}\left(1-2 c_{\mathrm{W}}^{2}\right)\left[s\left(s+M_{\mathrm{H}}^{2}\right)\left(1-4 c_{\mathrm{W}}^{2}\right)+2 c_{\mathrm{W}}^{2} M_{\mathrm{H}}^{4}\right]}{2 c_{\mathrm{W}}^{2} s_{\mathrm{W}}^{2} s^{2}} C_{\mathrm{HWZ}}^{h} \\
& -\frac{s\left(1-2 c_{\mathrm{W}}^{2}\right)}{s_{\mathrm{W}}^{2}}\left(C_{\mathrm{HWZ}}^{h}-C_{\mathrm{HWZ}}^{s}\right)-\frac{c_{\mathrm{W}}^{2} M_{\mathrm{H}}^{4}\left(2 M_{\mathrm{H}}^{2}+s\right)}{s_{\mathrm{W}}^{2} s t} C_{\mathrm{WHW}}^{h} \\
& -\frac{M_{\mathrm{H}}^{2}\left[2 c_{\mathrm{W}}^{2} s^{2}+s M_{\mathrm{H}}^{2}\left(1-2 c_{\mathrm{W}}^{2}+8 c_{\mathrm{W}}^{4}\right)+2\left(1+8 c_{\mathrm{W}}^{4}\right) M_{\mathrm{H}}^{4}\right]}{2 c_{\mathrm{W}}^{2} s_{\mathrm{W}}^{2} s^{2}} C_{\mathrm{WHW}}^{h} \\
& +\frac{M_{\mathrm{H}}^{4}\left(2 M_{\mathrm{H}}^{2}+s\right)}{4 s_{\mathrm{W}}^{2} s M_{\mathrm{W}}^{2}}\left(C_{\mathrm{WHW}}^{h}-C_{\mathrm{WHW}}^{h 1}\right)+\frac{c_{\mathrm{W}}^{2} s(2 u+s)}{2 s_{\mathrm{W}}^{2} u}\left(C_{\mathrm{WHW}}^{h}-C_{\mathrm{WHW}}^{s}\right) \\
& \left.+\frac{c_{\mathrm{W}}^{2} s t}{s_{\mathrm{W}}^{2} u}\left(C_{\nu \mathrm{WH}}^{h}-C_{\nu \mathrm{WH}}^{s}\right)-\frac{c_{\mathrm{W}}^{2} s(2 u+s)}{2 s_{\mathrm{W}}^{2} u}\left(D_{\mathrm{W} \nu \mathrm{WH}}^{h}\left(t-M_{\mathrm{H}}^{2}\right)-t D_{\mathrm{W} \nu \mathrm{WH}}^{s}\right)\right], \\
& C_{+, L}^{B, 2}=\frac{\alpha}{2 \pi}\left[\frac{M_{\mathrm{H}}^{2}\left[s\left(23-34 c_{\mathrm{W}}^{2}\right)-2 M_{\mathrm{H}}^{2}\left(13+22 c_{\mathrm{W}}^{2}\right)\right]}{24 c_{\mathrm{W}}^{2} s_{\mathrm{W}}^{2} s^{2}}+\frac{M_{\mathrm{H}}^{4}}{2 c_{\mathrm{W}}^{2} s_{\mathrm{W}}^{2} s^{2}} L_{\mathrm{Z}}+\frac{M_{\mathrm{H}}^{2}\left(M_{\mathrm{H}}^{2}-s\right)}{2 c_{\mathrm{W}}^{2} s_{\mathrm{W}}^{2} s^{2}} \beta_{\mathrm{Z}} L_{x_{\mathrm{Z}}}\right. \\
& -\frac{M_{\mathrm{H}}^{2}\left[s\left(1-2 c_{\mathrm{W}}^{2}\right)+4 c_{\mathrm{W}}^{2} M_{\mathrm{H}}^{2}\right]}{2 c_{\mathrm{W}}^{2} s_{\mathrm{W}}^{2} s^{2}} L_{s}+\frac{M_{\mathrm{H}}^{2}\left[8 s\left(1-2 c_{\mathrm{W}}^{2}\right)-M_{\mathrm{H}}^{2}\left(13+10 c_{\mathrm{W}}^{2}\right)\right]}{12 c_{\mathrm{W}}^{2} s_{\mathrm{W}}^{2} s^{2}}\left(L_{\mathrm{H}}-L_{s}\right) \\
& -\frac{s^{2}\left(1+22 c_{\mathrm{W}}^{2}\right)-14 s M_{\mathrm{H}}^{2}\left(1-2 c_{\mathrm{W}}^{2}\right)+M_{\mathrm{H}}^{4}\left(1-26 c_{\mathrm{W}}^{2}\right)}{12 c_{\mathrm{W}}^{2} s_{\mathrm{W}}^{2} s^{2}}\left(B_{\mathrm{HZ}}-B^{s}\right) \\
& -\frac{M_{\mathrm{H}}^{2}\left[s\left(s+M_{\mathrm{H}}^{2}\right)\left(1-4 c_{\mathrm{W}}^{2}\right)+2 c_{\mathrm{W}}^{2} M_{\mathrm{H}}^{4}\right]}{2 c_{\mathrm{W}}^{2} s_{\mathrm{W}}^{2} s^{2}} C_{\mathrm{HWZ}}^{h}+\frac{s}{s_{\mathrm{W}}^{2}}\left(C_{\mathrm{HWZ}}^{s}-C_{\mathrm{HWZ}}^{h}+\frac{L_{x_{\mathrm{H}}}^{2}}{2 s}-\frac{L_{\mathrm{H}}^{2}}{2 s}\right) \\
& \left.-\frac{M_{\mathrm{H}}^{2}\left[2 c_{\mathrm{W}}^{2} s^{2}+s M_{\mathrm{H}}^{2}+2\left(1+2 c_{\mathrm{W}}^{2}\right) M_{\mathrm{H}}^{4}\right]}{2 c_{\mathrm{W}}^{2} s_{\mathrm{W}}^{2} s^{2}} C_{\mathrm{WHW}}^{h}+\frac{M_{\mathrm{H}}^{4}\left(2 M_{\mathrm{H}}^{2}+s\right)}{4 s_{\mathrm{W}}^{2} s M_{\mathrm{W}}^{2}}\left(C_{\mathrm{WHW}}^{h}-C_{\mathrm{WHW}}^{h 1}\right)\right] \text {, }
\end{aligned}
$$$$
C_{-. T}^{B, 2}=0,
$$ 


$$
\begin{aligned}
& C_{-, L}^{F, 2}=-2 \frac{M_{\mathrm{W}}^{2}}{s} \frac{c_{\mathrm{W}}^{2}}{s_{\mathrm{W}}^{2}} \Delta \rho\left(24 c_{\mathrm{W}}^{4}+4 s_{\mathrm{W}}^{2}-\frac{2}{c_{\mathrm{W}}^{2}}+8 c_{\mathrm{W}}^{4} \frac{s}{t}\right)+\frac{\alpha}{2 \pi}\left[\frac{6 c_{\mathrm{W}}^{2} m_{\mathrm{t}}^{2}}{t}\right. \\
& +\frac{\left(47-160 c_{\mathrm{W}}^{2}+392 c_{\mathrm{W}}^{4}-324 c_{\mathrm{W}}^{6}\right) m_{\mathrm{t}}^{2}}{18 c_{\mathrm{W}}^{2} s_{\mathrm{W}}^{2} s}+\frac{6 c_{\mathrm{W}}^{2}\left(s+4 c_{\mathrm{W}}^{2} m_{\mathrm{t}}^{2}\right) m_{\mathrm{t}}^{2}}{s_{\mathrm{W}}^{2} s t}\left(L_{\mathrm{top}}-L_{s}+1\right) \\
& +\frac{m_{\mathrm{t}}^{2}\left[s\left(47-286 c_{\mathrm{W}}^{2}+572 c_{\mathrm{W}}^{4}\right)+648 c_{\mathrm{W}}^{4}\left(1+2 c_{\mathrm{W}}^{2}\right) m_{\mathrm{t}}^{2}\right]}{18 c_{\mathrm{W}}^{2} s_{\mathrm{W}}^{2} s^{2}}\left(L_{\mathrm{top}}-L_{s}+1\right) \\
& +\left(B_{\mathrm{tt}}-B^{s}\right)\left\{\frac{4 c_{\mathrm{W}}^{2} m_{\mathrm{t}}^{2}\left[2 s_{\mathrm{W}}^{2} s+\left(1+2 c_{\mathrm{W}}^{2}\right) m_{\mathrm{t}}^{2}\right]}{s_{\mathrm{W}}^{2} s t}-\frac{17-28 c_{\mathrm{W}}^{2}+20 c_{\mathrm{W}}^{4}}{18 c_{\mathrm{W}}^{2} s_{\mathrm{W}}^{2}}\right. \\
& \left.+\frac{m_{\mathrm{t}}^{2}\left(65-304 c_{\mathrm{W}}^{2}+572 c_{\mathrm{W}}^{4}-432 c_{\mathrm{W}}^{6}\right)}{18 c_{\mathrm{W}}^{2} s_{\mathrm{W}}^{2} s}+\frac{2 m_{\mathrm{t}}^{4}\left(1+14 c_{\mathrm{W}}^{4}+12 c_{\mathrm{W}}^{6}\right)}{c_{\mathrm{W}}^{2} s_{\mathrm{W}}^{2} s^{2}}\right\} \\
& -\left(\frac{4 c_{\mathrm{W}}^{2}\left(1-4 c_{\mathrm{W}}^{2}\right)\left(s+m_{\mathrm{t}}^{2}\right)}{s_{\mathrm{W}}^{2} t}+\frac{2\left[s\left(s_{\mathrm{W}}^{2}-24 c_{\mathrm{W}}^{6}\right)+m_{\mathrm{t}}^{2}\left(1-4 c_{\mathrm{W}}^{4}-24 c_{\mathrm{W}}^{6}\right)\right]}{c_{\mathrm{W}}^{2} s_{\mathrm{W}}^{2} s}\right) \frac{m_{\mathrm{t}}^{4}}{s} C_{\mathrm{btb}}^{h} \\
& -\frac{4 c_{\mathrm{W}}^{2}\left(1+2 c_{\mathrm{W}}^{2}\right) m_{\mathrm{t}}^{6}}{s_{\mathrm{W}}^{2} s t} C_{\mathrm{tbt}}^{h}+\frac{m_{\mathrm{t}}^{4}\left[c_{\mathrm{W}}^{2} s\left(7+2 c_{\mathrm{W}}^{2}\right)-2 m_{\mathrm{t}}^{2}\left(1+14 c_{\mathrm{W}}^{4}+12 c_{\mathrm{W}}^{6}\right)\right]}{c_{\mathrm{W}}^{2} s_{\mathrm{W}}^{2} s^{2}} C_{\mathrm{tbt}}^{h} \\
& \left.-\frac{\left(1-4 c_{\mathrm{W}}^{2}\right) m_{\mathrm{t}}^{4}}{s_{\mathrm{W}}^{2} M_{\mathrm{W}}^{2}}\left(1+\frac{m_{\mathrm{t}}^{2}}{s}\right)\left(C_{\mathrm{btb}}^{h 1}-C_{\mathrm{btb}}^{h}\right)-\frac{\left(1+2 c_{\mathrm{W}}^{2}\right) m_{\mathrm{t}}^{6}}{s_{\mathrm{W}}^{2} s M_{\mathrm{W}}^{2}}\left(C_{\mathrm{tbt}}^{h 1}-C_{\mathrm{tbt}}^{h}\right)\right], \\
& C_{+, L}^{F, 2}=\frac{\alpha}{2 \pi}\left[\frac{\left(47+c_{\mathrm{W}}^{2}\right) m_{\mathrm{t}}^{2}}{18 c_{\mathrm{W}}^{2} s_{\mathrm{W}}^{2} s}+\frac{m_{\mathrm{t}}^{2}\left[s\left(47-152 c_{\mathrm{W}}^{2}\right)-108 c_{\mathrm{W}}^{2} m_{\mathrm{t}}^{2}\right]}{18 c_{\mathrm{W}}^{2} s_{\mathrm{W}}^{2} s^{2}}\left(L_{\mathrm{top}}-L_{s}+1\right)\right. \\
& -\frac{\left(17-14 c_{\mathrm{W}}^{2}\right) s^{2}-\left(65-98 c_{\mathrm{W}}^{2}\right) s m_{\mathrm{t}}^{2}-36\left(1+c_{\mathrm{W}}^{2}\right) m_{\mathrm{t}}^{4}}{18 c_{\mathrm{W}}^{2} s_{\mathrm{W}}^{2} s^{2}}\left(B_{\mathrm{tt}}-B^{s}\right) \\
& -\frac{2 m_{\mathrm{t}}^{4}\left[s\left(1+3 c_{\mathrm{W}}^{2}\right)+m_{\mathrm{t}}^{2}\left(1+4 c_{\mathrm{W}}^{2}\right)\right]}{c_{\mathrm{W}}^{2} s_{\mathrm{W}}^{2} s^{2}} C_{\mathrm{btb}}^{h}+\frac{m_{\mathrm{t}}^{4}\left[7 c_{\mathrm{W}}^{2} s-2 m_{\mathrm{t}}^{2}\left(1+c_{\mathrm{W}}^{2}\right)\right]}{c_{\mathrm{W}}^{2} s_{\mathrm{W}}^{2} s^{2}} C_{\mathrm{tbt}}^{h} \\
& \left.-\frac{m_{\mathrm{t}}^{4}}{s_{\mathrm{W}}^{2} M_{\mathrm{W}}^{2}}\left(1+\frac{m_{\mathrm{t}}^{2}}{s}\right)\left(C_{\mathrm{btb}}^{h 1}-C_{\mathrm{btb}}^{h}\right)-\frac{m_{\mathrm{t}}^{6}}{s_{\mathrm{W}}^{2} s M_{\mathrm{W}}^{2}}\left(C_{\mathrm{tbt}}^{h 1}-C_{\mathrm{tbt}}^{h}\right)\right], \\
& C_{-, T}^{F, 2}=-8 \frac{M_{\mathrm{W}}^{2}}{s} \frac{c_{\mathrm{W}}^{2}}{s_{\mathrm{W}}^{2}} \Delta \rho \\
& \left(\frac{d \sigma}{d \Omega}\right)_{-, M}^{B}=F \frac{\alpha}{2 \pi} \frac{M_{\mathrm{H}}^{2}}{8 c_{\mathrm{W}}^{4} s_{\mathrm{W}}^{6} s} \frac{u^{2}+t^{2}+2 c_{\mathrm{W}}^{2} u(u-t)}{s^{2}} \\
& \times\left\{s_{\mathrm{W}}^{2}+\left[\frac{M_{\mathrm{H}}^{2}}{s}\left(1+2 c_{\mathrm{W}}^{2}\right)+2 s_{\mathrm{W}}^{2}\right]\left(L_{\mathrm{H}}-L_{s}+1\right)\right. \\
& \left.+\left(1-2 c_{\mathrm{W}}^{2}\right)\left(1-\frac{M_{\mathrm{H}}^{2}}{s}\right)\left(B_{\mathrm{HZ}}-B^{s}\right)+2 M_{\mathrm{H}}^{2}\left(1+\frac{M_{\mathrm{H}}^{2}}{s}\right) C_{\mathrm{WHW}}^{h}\right\}, \\
& \left(\frac{d \sigma}{d \Omega}\right)_{+, M}^{B}=F \frac{\alpha}{2 \pi} \frac{M_{\mathrm{H}}^{2}}{2 c_{\mathrm{W}}^{4} s_{\mathrm{W}}^{2} s} \frac{u^{2}+t^{2}}{s^{2}}\left\{1+\left[\frac{M_{\mathrm{H}}^{2}}{s}+2\right]\left(L_{\mathrm{H}}-L_{s}+1\right)\right. \\
& \left.+\left(1-\frac{M_{\mathrm{H}}^{2}}{s}\right)\left(B_{\mathrm{HZ}}-B^{s}\right)+2 M_{\mathrm{H}}^{2}\left(1+\frac{M_{\mathrm{H}}^{2}}{s}\right) C_{\mathrm{WHW}}^{h}\right\},
\end{aligned}
$$




$$
\begin{aligned}
\left(\frac{d \sigma}{d \Omega}\right)_{-, M}^{F}= & F \frac{2 M_{\mathrm{W}}^{2}}{s} \frac{\Delta \rho}{c_{\mathrm{W}}^{2} s_{\mathrm{W}}^{6}} \frac{\left(1-2 c_{\mathrm{W}}^{2}\right)\left(u^{2}+t^{2}\right)-4 c_{\mathrm{W}}^{4} u(3 u-t)}{s^{2}} \\
+ & F \frac{\alpha}{2 \pi} \frac{m_{\mathrm{t}}^{2}}{4 c_{\mathrm{W}}^{4} s_{\mathrm{W}}^{6} s}\left\{\left(8-11 c_{\mathrm{W}}^{2}\right) \frac{u^{2}+t^{2}+2 c_{\mathrm{W}}^{2} u(u-t)}{s^{2}}\right. \\
& +2\left[\frac{u^{2}+4 t^{2}-c_{\mathrm{W}}^{2}\left(5 u^{2}+8 u t+t^{2}\right)-2 c_{\mathrm{W}}^{4} u(7 u-t)}{s^{2}}\right. \\
& \left.-6 \frac{m_{\mathrm{t}}^{2} c_{\mathrm{W}}^{2}\left[u^{2}+t^{2}+2 c_{\mathrm{W}}^{2} u(u-t)\right]}{s^{3}}\right]\left(L_{\mathrm{top}}-L_{s}+1\right) \\
& {\left[\frac{u^{2}-9 t^{2}+c_{\mathrm{W}}^{2}\left(4 u^{2}+18 u t+6 t^{2}\right)+4 c_{\mathrm{W}}^{4} u(u-3 t)}{s^{2}}\right.} \\
& +2\left(1-4 c_{\mathrm{W}}^{2}\right) \frac{\left(1+2 c_{\mathrm{W}}^{2}\right) s u^{2}\left(s+2 m_{\mathrm{t}}^{2}\right)+m_{\mathrm{t}}^{4}\left[u^{2}+t^{2}+2 c_{\mathrm{W}}^{2} u(u-t)\right]}{s^{3}} C_{\mathrm{btb}}^{h} \\
& +2\left(1+2 c_{\mathrm{W}}^{2}\right) m_{\mathrm{t}}^{2}\left[\frac{4 u^{2}-t^{2}-2 c_{\mathrm{W}}^{2} u(2 u-t)}{s^{2}}\right. \\
& \left.\left.+\frac{m_{\mathrm{t}}^{2}\left[u^{2}+t^{2}+2 c^{2}+t^{2}+2 c_{\mathrm{W}}^{2} u(u-t)\right]}{s^{3}}\right] C_{\mathrm{tbt}}^{h}\right\} \\
\left(\frac{d \sigma}{d \Omega}\right)_{+, M}^{F}=F \frac{\alpha}{2 \pi} & \frac{m_{\mathrm{t}}^{2}}{c_{\mathrm{W}}^{4} s_{\mathrm{W}}^{2} s}\left\{8 \frac{u^{2}+t^{2}}{s^{2}}+2 \frac{4 u^{2}+t^{2}}{s^{2}}\left(L_{\mathrm{top}}-L_{s}+1\right)\right. \\
+ & \frac{s\left(9 u^{2}-t^{2}\right)-2 m_{\mathrm{t}}^{2}\left(u^{2}+t^{2}\right)}{s^{3}}\left(B_{\mathrm{tt}}-B^{s}\right) \\
+ & 2 \frac{s^{2} t^{2}+2 s t^{2} m_{\mathrm{t}}^{2}+m_{\mathrm{t}}^{4}\left(u^{2}+t^{2}\right)}{s^{3}} C_{\mathrm{btb}}^{h} \\
& 2 m_{\mathrm{t}}^{2} \frac{s\left(u^{2}-4 t^{2}\right)-m_{\mathrm{t}}^{2}\left(u^{2}+t^{2}\right)}{\left.s_{\mathrm{tbt}}^{h}\right\} \cdot}
\end{aligned}
$$

The various asymptotic scalar integrals, like $C_{\mathrm{WHW}}^{h}$, can be found in Appendix A. All other abbreviations have been defined in (13) and (A.2).

\section{References}

[1] Physics at LEP, CERN 86-02, eds. J. Ellis and R. Peccei, Vol. 2;

ECFA Workshop on LEPQ00, CERN 87-08, eds. A. Böhm and W. Hoogland, Vol. 1; K. Hagiwara, R.D. Peccei, D. Zeppenfeld and K. Hikasa, Nucl. Phys. B282 (1987) 253 ;

W. Beenakker, F.A. Berends and A. Denner, in : Proceedings of the Workshop on $\mathrm{e}^{+} \mathrm{e}^{-}$-collisions at $500 \mathrm{GeV}$ : The Physics Potential, Munich, Annecy, Hamburg, 1991, ed. P. Zerwas, DESY 92-123A (Hamburg, 1992) p. 151.

[2] M. Böhm, A. Denner, T. Sack, W. Beenakker, F.A. Berends and H. Kuijf, Nucl. Phys. B304 (1988) 463.

[3] J. Fleischer, F. Jegerlehner and M. Zrałek, Z. Phys. C42 (1989) 409. 
[4] W. Beenakker, K. Kołodziej and T. Sack, Phys. Lett. B258 (1991) 469;

W. Beenakker, F.A. Berends and T. Sack, Nucl. Phys. B367 (1991) 287.

[5] K. Kołodziej and M. Zrałek, Phys. Rev. D43 (1991) 3619;

J. Fleischer, F. Jegerlehner and K. Kołodziej, Phys. Rev. D47 (1993) 830.

[6] S. Dittmaier, M. Böhm and A. Denner, Nucl. Phys. B376 (1992) 29.

[7] C. Ahn, B.W. Lynn, M. Peskin and S. Selipsky, Nucl. Phys. B309 (1988) 221.

[8] S. Wolfram, Mathematica: A System for Doing Mathematics by Computer (AddisonWesley, Redwood City, CA, 1991) Second Edition.

[9] J.A.M. Vermaseren, Symbolic Manipulation with FORM (Computer Algebra Nederland, Amsterdam, 1991).

[10] J.M. Cornwall, D.N. Levin and G. Tiktopoulos, Phys. Rev. D10 (1974) 1145;

B.W. Lee, C. Quigg and H. Thacker, Phys. Rev. D16 (1977) 1519;

M.S. Chanowitz and M.K. Gaillard, Nucl. Phys. B261 (1985) 379;

H. Veltman, Phys. Rev. D41 (1990) 2294.

[11] M. Lemoine and M. Veltman, Nucl. Phys. B164 (1980) 445.

[12] A. Denner, Habilitation Thesis, University of Würzburg 1991, to appear in Fortschr. Phys. 41 (1993).

[13] W. Beenakker, A. Denner, S. Dittmaier and R. Mertig, in preparation.

[14] F. Jegerlehner, in Proc. 1990 Theoretical Advanced Study Institute in Elementary Particle Physics, eds. M. Cvetič and P. Langacker (World Scientific, Singapore, 1991), p. 476. 


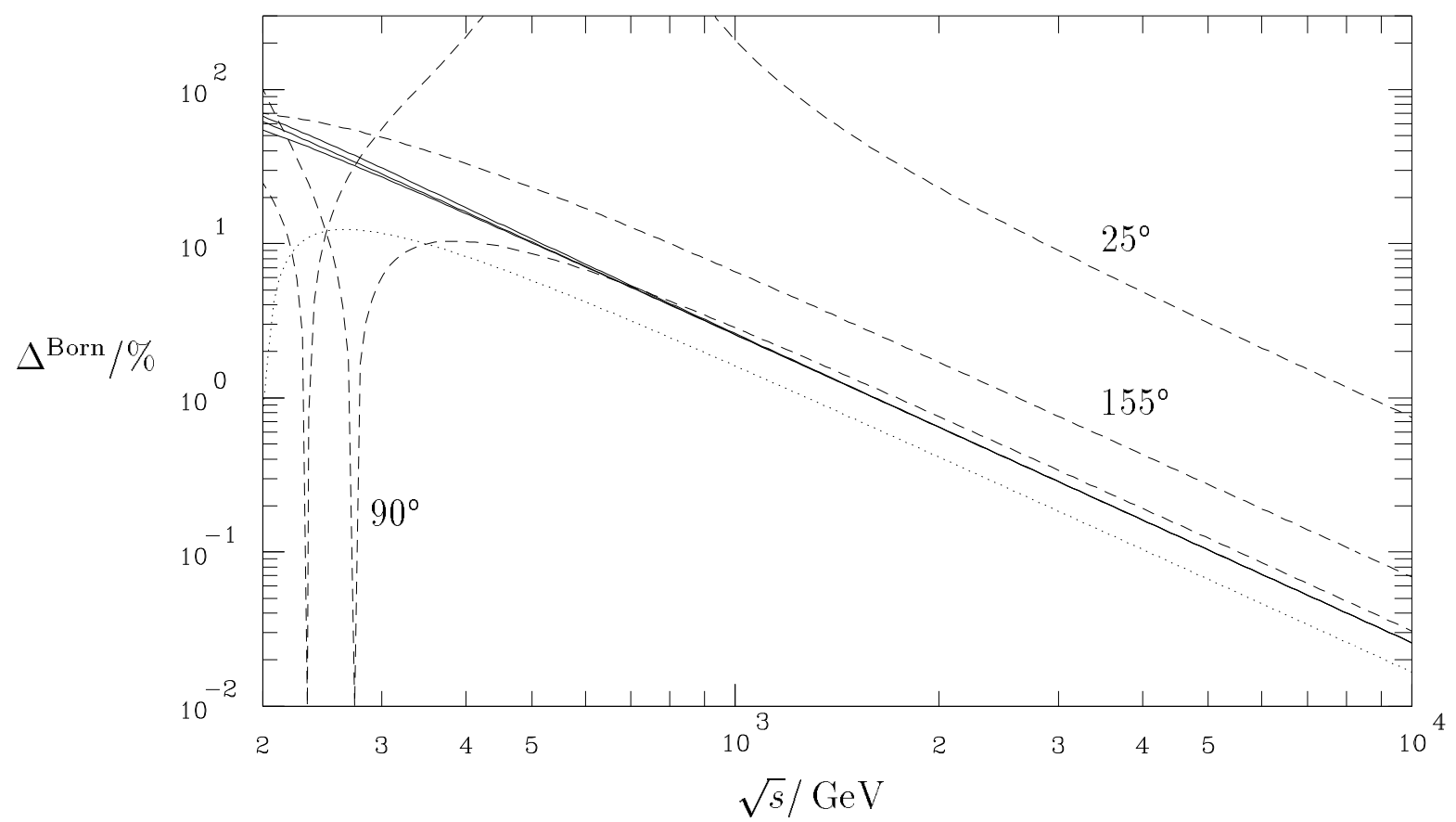

Figure 1: Deviation of the lowest-order approximations from the full Born cross-sections: $(-, L):(---),(+, L):(\cdots \cdots)$ and $(-, T):(-)$.

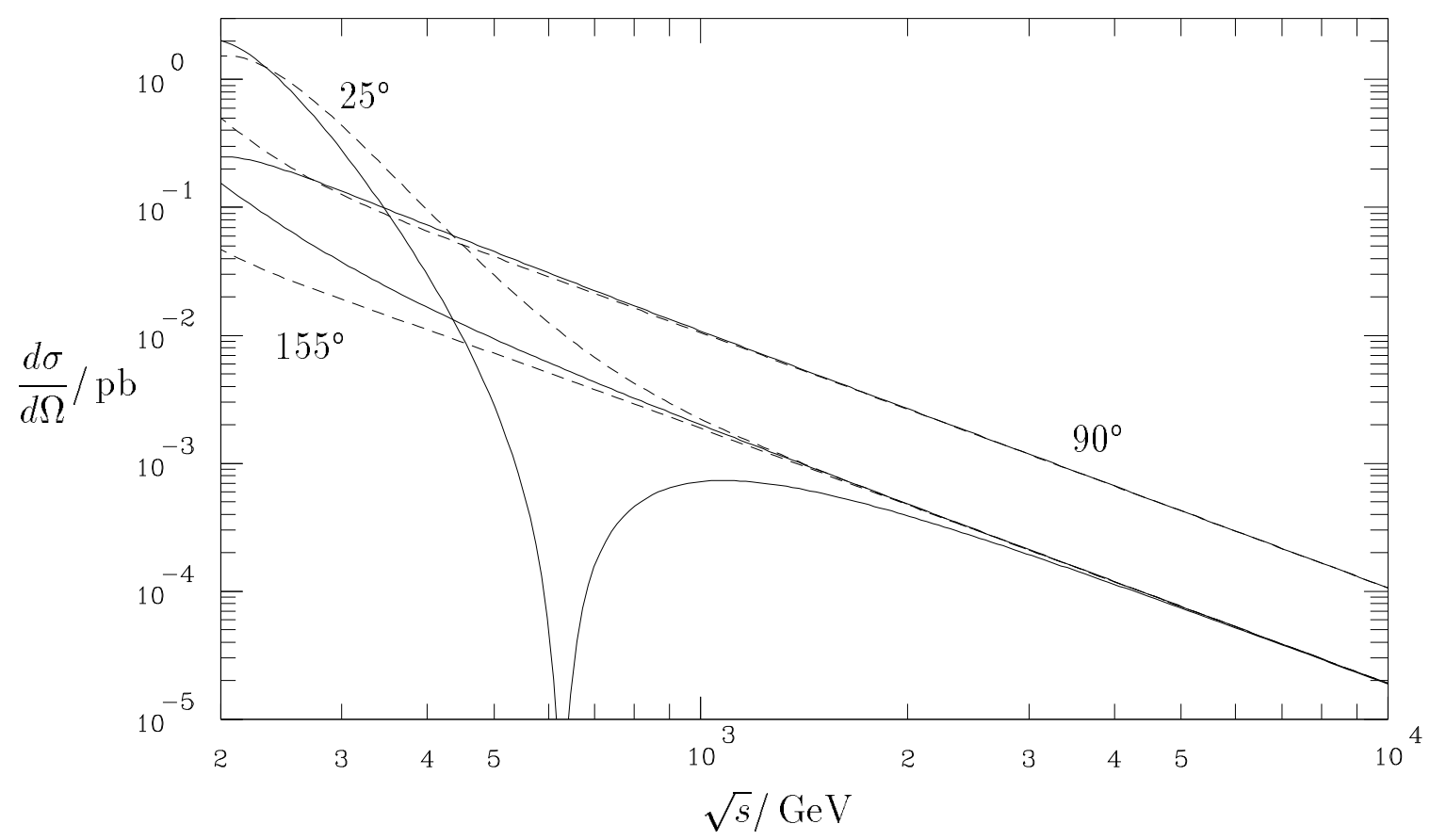

Figure 2: Lowest-order cross-section for $(-, L)(-)$ and the corresponding approximation (24) (- - - ) for different scattering angles. 

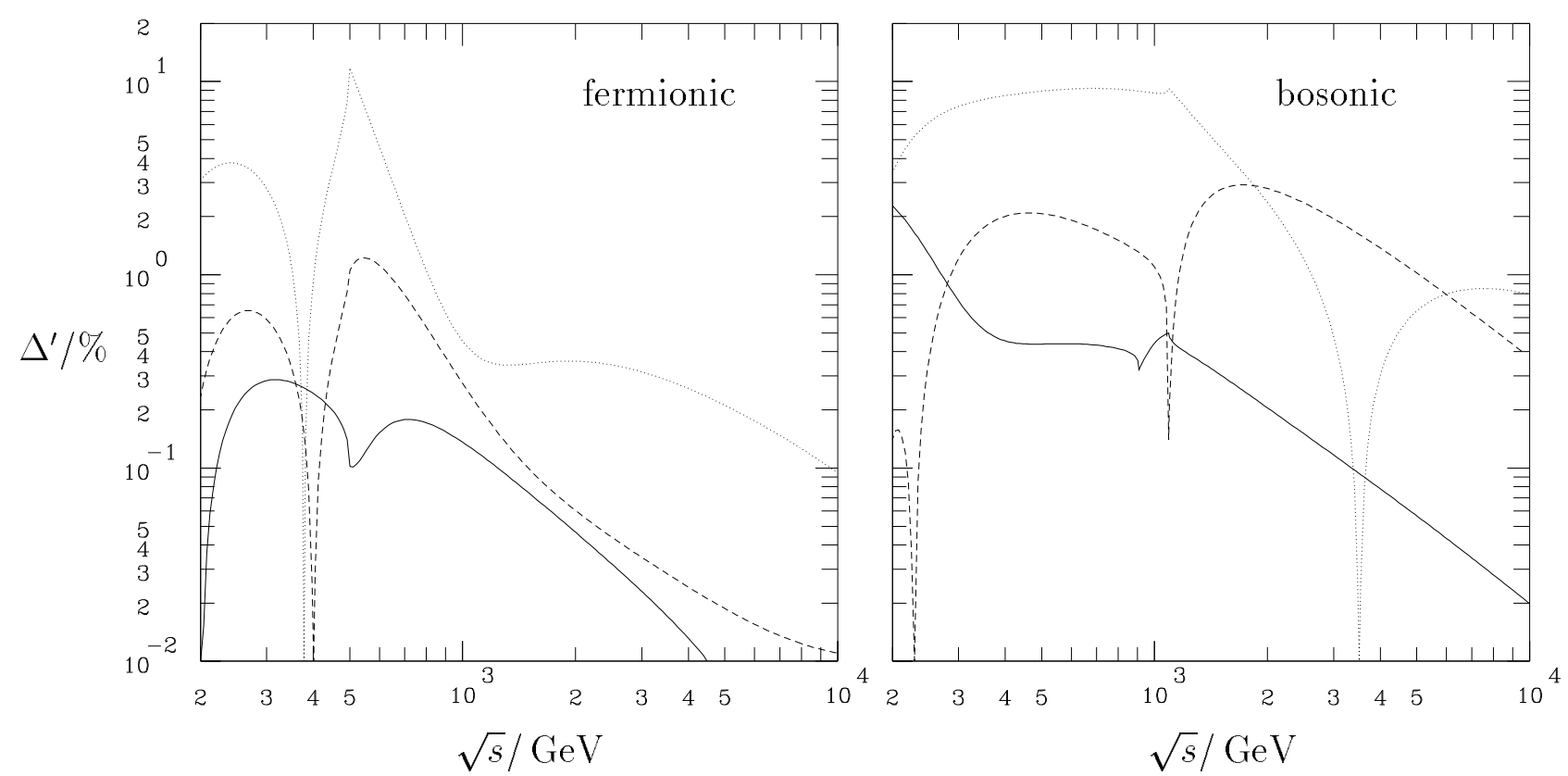

Figure 3: Quality of the approximations for $(-, L)$ resulting from $C^{0}(\cdots \cdots), C^{0}+C^{1}$ $(---)$ and $C^{0}+C^{1}+C^{2}(-)$ for $\theta=90^{\circ}$.
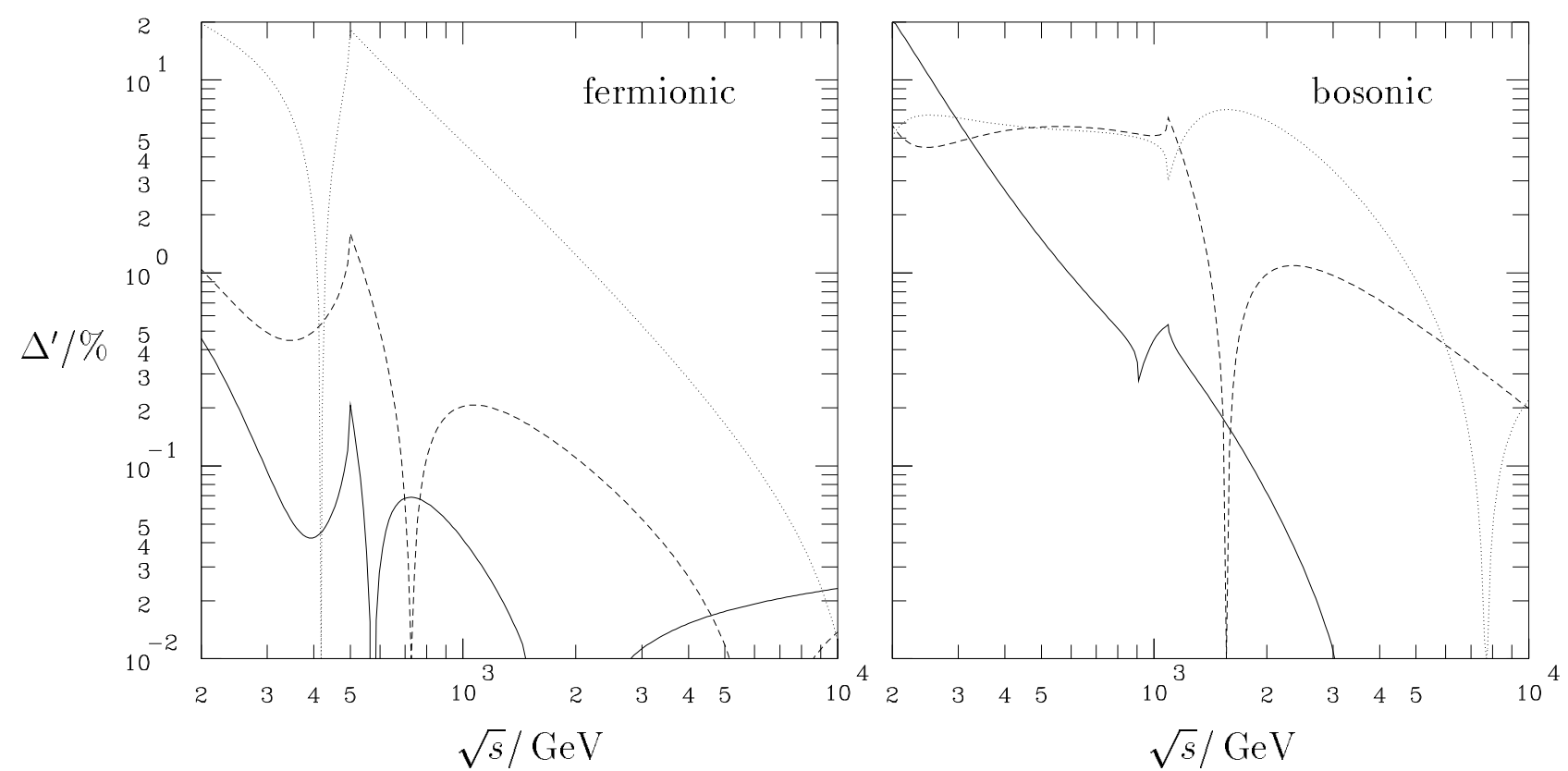

Figure 4: Quality of the approximations for $(+, L)$ resulting from $C^{0}(\cdots \cdots), C^{0}+C^{1}$ $(---)$ and $C^{0}+C^{1}+C^{2}(-)$ for $\theta=90^{\circ}$. 

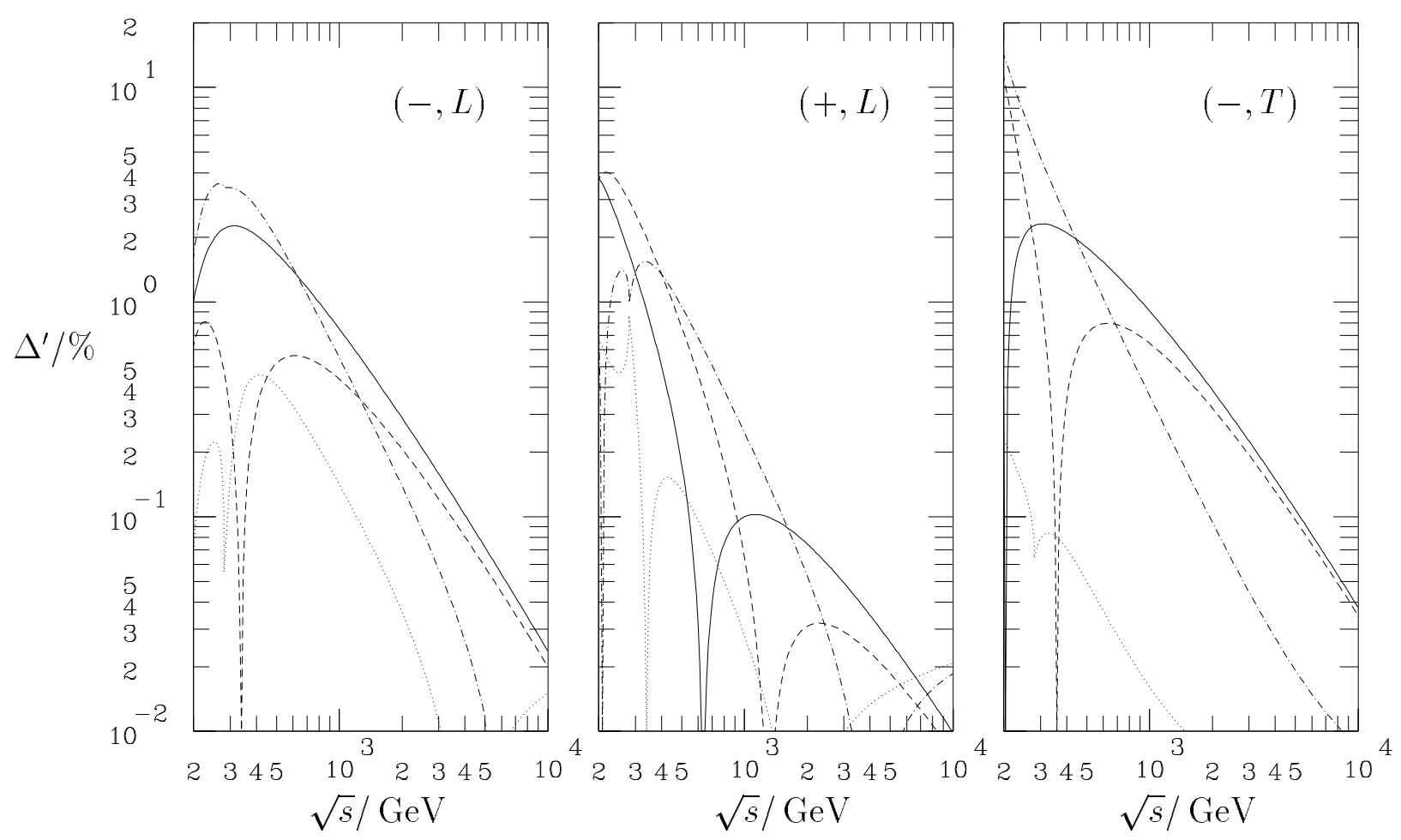

Figure 5: Quality of the approximations with $\left(C^{B}\right.$ : and $\left.C^{F}: \cdots \cdots\right)$ and without $\left(C^{B}:---\right.$ and $\left.C^{F}:-\cdot-\cdot\right)$ the improvement for the contributions associated with $\Delta \alpha$, $\Delta \rho$ and $\Delta_{\mathrm{LL}}\left(\theta=90^{\circ}\right)$.

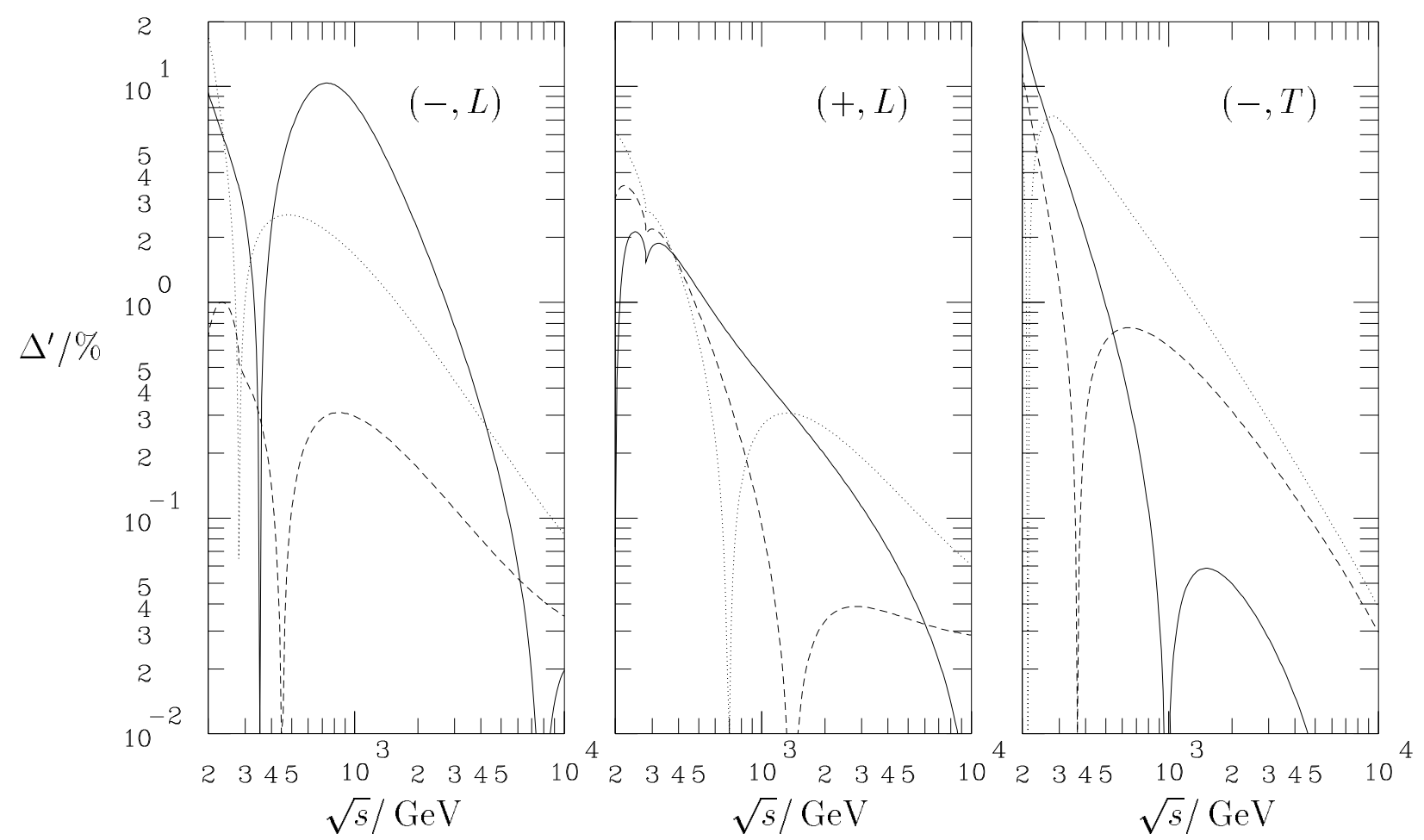

Figure 6: Quality of the approximations as a function of the energy for the scattering angles $25^{\circ}(-), 90^{\circ}(---)$ and $155^{\circ}(\cdots \cdots)$. 

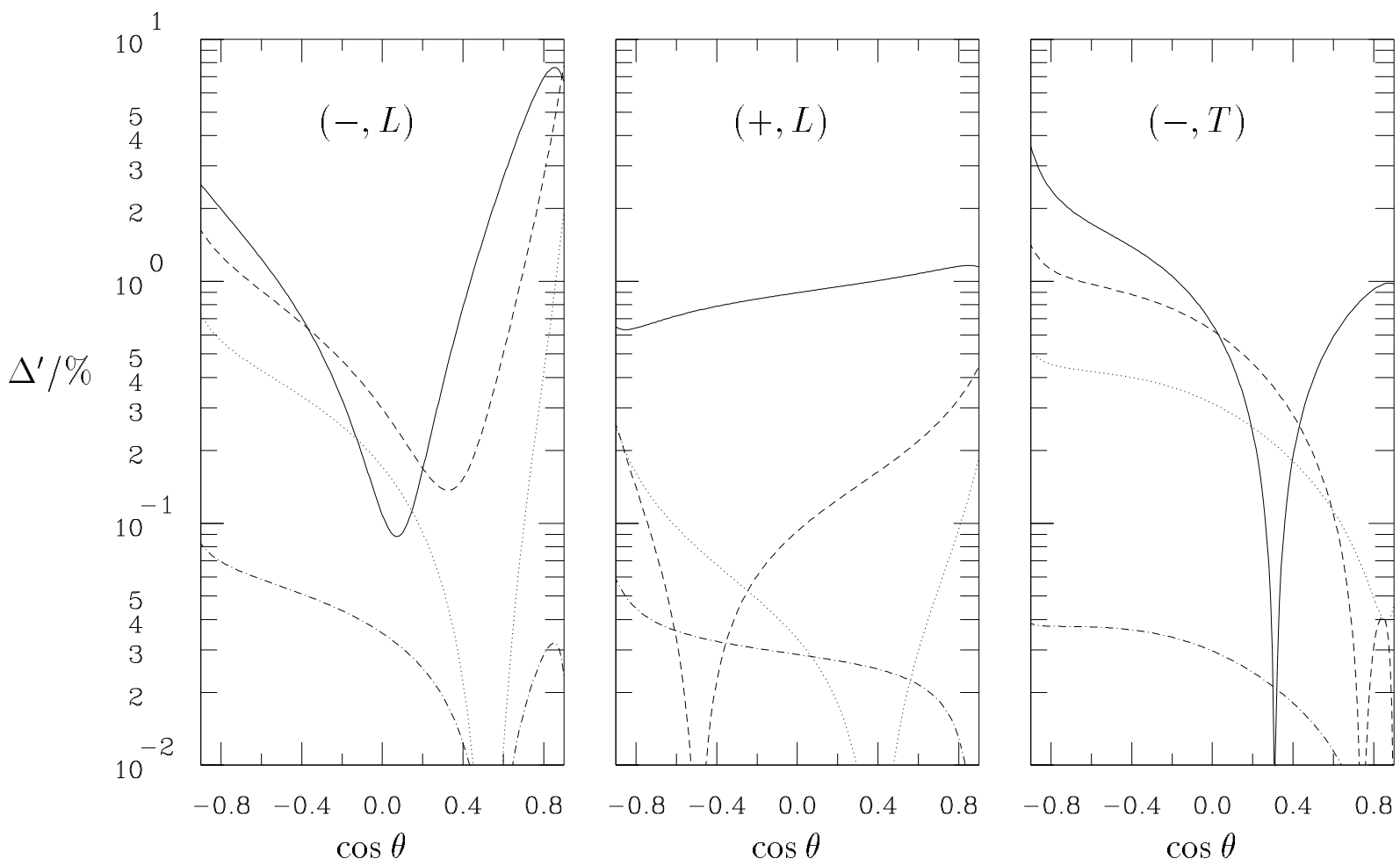

Figure 7: Quality of the approximations as a function of the scattering angle for the energies $500 \mathrm{GeV}(\longrightarrow), 1 \mathrm{TeV}(---), 2 \mathrm{TeV}(\cdots \cdots)$ ) and $10 \mathrm{TeV}(-\cdot-\cdot)$.

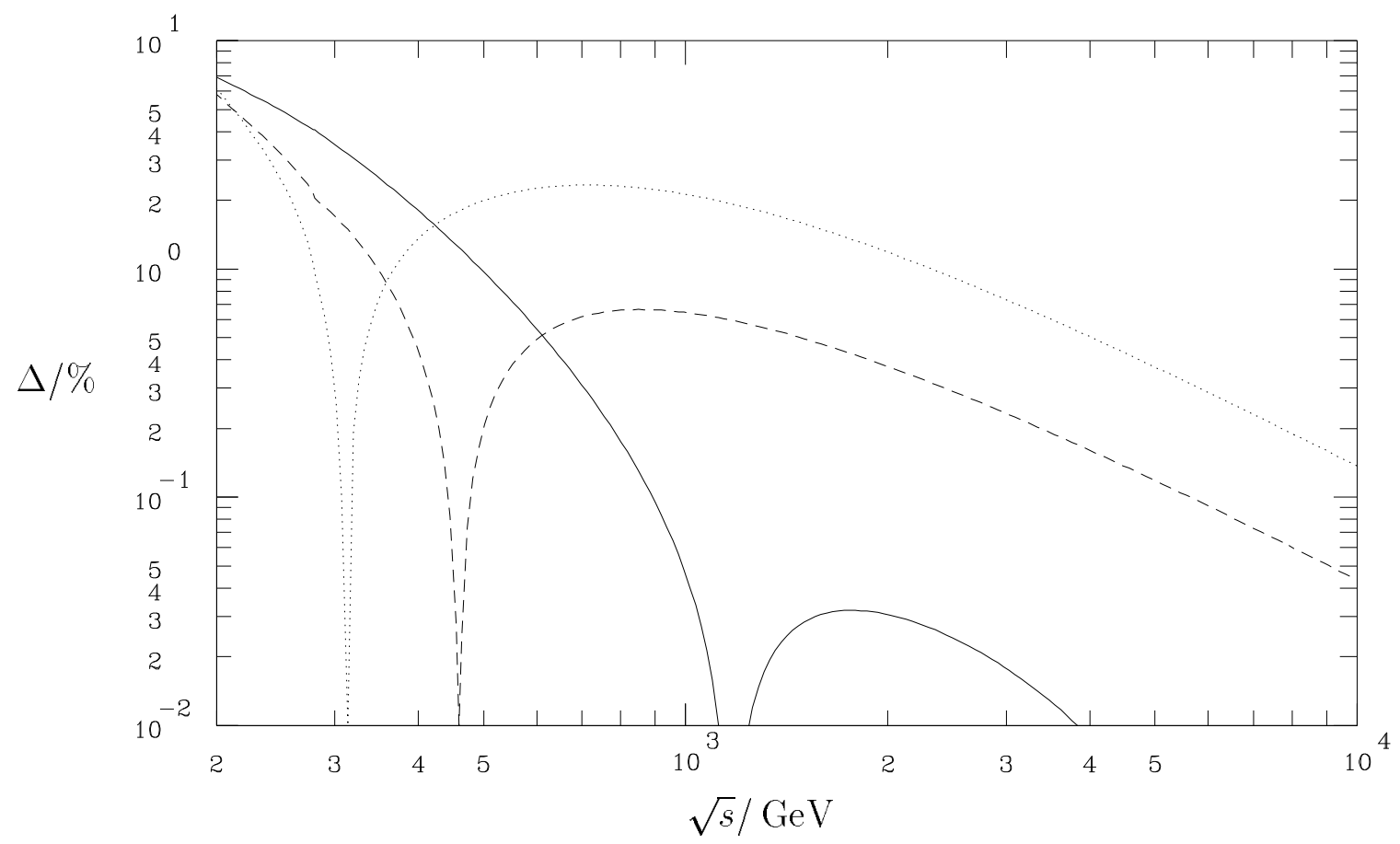

Figure 8: Quality of the approximation for the unpolarized cross-section as a function of the energy for the scattering angles $25^{\circ}(-), 90^{\circ}(---)$ and $155^{\circ}(\cdots \cdots)$. 


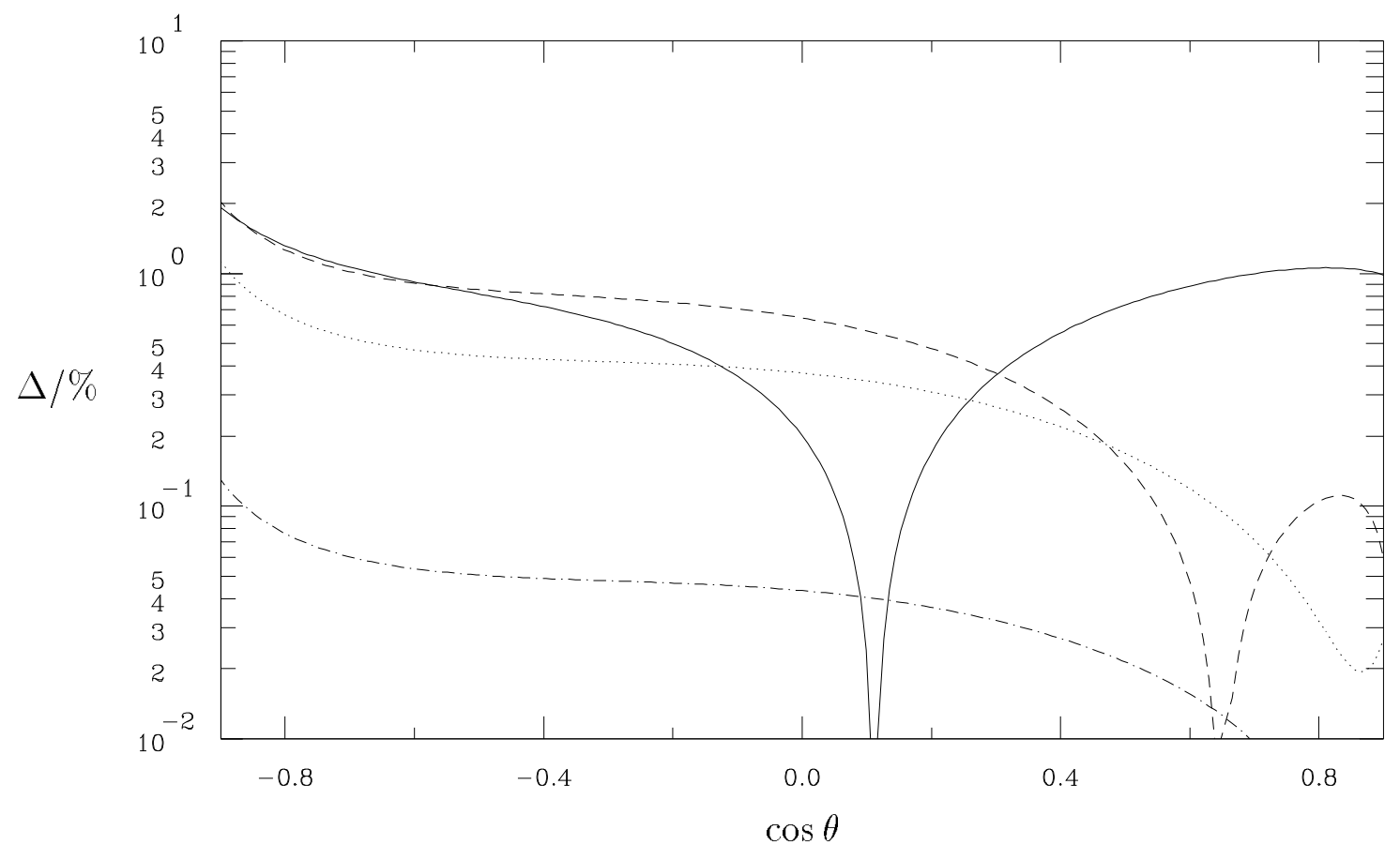

Figure 9: Quality of the approximation for the unpolarized cross-section as a function of the scattering angle for the energies $500 \mathrm{GeV}(-), 1 \mathrm{TeV}(---), 2 \mathrm{TeV}(\cdots \cdots)$ and $10 \mathrm{TeV}(-\cdot-\cdot)$.

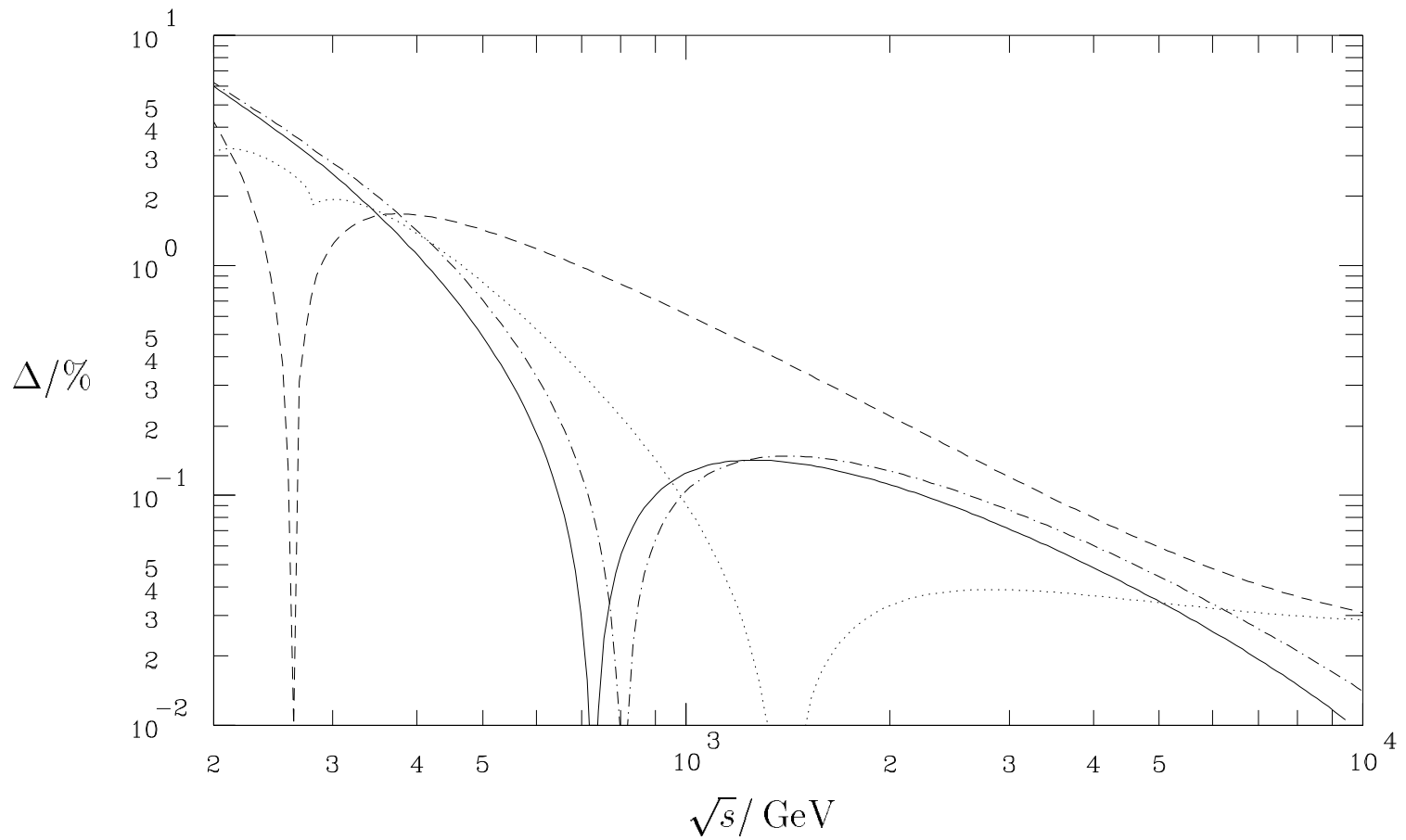

Figure 10: Quality of the approximations for the integrated cross-sections $(-0.9<\cos \theta<$ $0.9):(-, L):(---),(+, L):(\cdots \cdots),(-, T):(-)$, and (unpol.): $(-\cdot-\cdot)$. 